\title{
Determining the Points of Change in Time Series of Polarimetric SAR Data
}

\author{
Conradsen, Knut; Nielsen, Allan Aasbjerg; Skriver, Henning
}

Published in:

IEEE Transactions on Geoscience and Remote Sensing

Link to article, DOI:

10.1109/TGRS.2015.2510160

Publication date:

2016

Document Version

Peer reviewed version

Link back to DTU Orbit

Citation (APA):

Conradsen, K., Nielsen, A. A., \& Skriver, H. (2016). Determining the Points of Change in Time Series of Polarimetric SAR Data. IEEE Transactions on Geoscience and Remote Sensing, 54(5), 3007-3024. https://doi.org/10.1109/TGRS.2015.2510160

\section{General rights}

Copyright and moral rights for the publications made accessible in the public portal are retained by the authors and/or other copyright owners and it is a condition of accessing publications that users recognise and abide by the legal requirements associated with these rights.

- Users may download and print one copy of any publication from the public portal for the purpose of private study or research.

- You may not further distribute the material or use it for any profit-making activity or commercial gain

- You may freely distribute the URL identifying the publication in the public portal 


\title{
Determining the Points of Change in Time Series of Polarimetric SAR Data
}

\author{
Knut Conradsen, Allan Aasbjerg Nielsen, and Henning Skriver, Member, IEEE
}

\begin{abstract}
We present the likelihood ratio test statistic for homogeneity of several complex variance-covariance matrices that may be used in order to assess whether at least one change has taken place in a time series of SAR data. We furthermore give a factorization of this test statistic into a product of test statistics that each tests simpler hypotheses of homogeneity up to a certain point and that are independent if the hypothesis of total homogeneity is true. This factorization is used in determining the (pixelwise) time points of change in a series of six L-band EMISAR polarimetric SAR data. The pixelwise analyses are applied on homogeneous subareas covered with different vegetation types using the distribution of the observed $p$-values.

http://www.imm.dtu.dk/pubdb/p.php?6825.
\end{abstract}

\section{Keywords}

Complex covariance matrix test statistic, complex Wishart distribution, omnibus test statistic, multi-temporal SAR data, quad polarization, full polarization, dual polarization, remote sensing change detection, EMISAR.

\section{INTRODUCTION}

$\mathrm{C}$ HANGE detection is a very important method for many applications of remotely sensed data from satellites. Especially SAR data are useful due to its all-weather capabilities, and hence planned acquisitions for change detection are normally secured. A number of studies have applied SAR data to change detection applications in single-channel SAR images applying different methods using e.g. the classical ratio detection [1], [2], the KittlerIllingworth threshold selection criterion [3]-[6], hidden Markov chains for thresholding [7], wavelets [8]-[11], linear features [12], Kullback-Leibler divergence [13], multivariate gamma distributions [14], neural networks [15], fusion of multi-similarity measures [16], and Markov random fields [17], where most of the methods are based on the classic ratio detector and improvements thereof. Also, methods for change detection using multichannel SAR data (e.g. polarimetric) have been studied using e.g. polarimetric parameters [18], [19], Markov random fields for multichannel SAR data [20], [21], generalized maximum likelihood test for covariance matrices [22] and the same test statistics for classification [23], partial vectors for suppression of the backscatter coefficient influence [24], the Hotelling-Lawley trace statistic [25], and a non-Wishart change detector [26], where a characteristic of the multichannel change detectors is that they are used for change detection between bi-temporal acquisitions.

In change detection between two polarimetric SAR images, tests comparing two complex variance-covariance matrices have turned out to be very efficient. This is used in e.g. [22] where the likelihood ratio test statistic is derived and an approximate expression for the distribution of the statistic under the hypothesis that no changes have occurred is found. In the radar literature the term "variance-covariance matrix" is not commonly used. We use the term to indicate that we here deal with quadratic, positive definite (dispersion) matrices and not cross-covariance matrices between different multivariate observations. In the paper, we apply the test statistics developed to multilook SAR data in the so-called complex covariance formulation. In this paper we will use the usual radar term "covariance matrix".

When comparing several images one may apply the simple approach making pairwise comparisons. However, this approach makes it virtually impossible to control the rates of false positives (postulating a change when there actually is none) and of false negatives (missing an actual change). In general, a better approach for comparing several distributions is to perform a simultaneous test of the hypothesis of homogeneity of the said distributions, a so-called omnibus test, see for example [27]. In this paper we enable this by deriving the likelihood ratio test statistic for equality of several, say $k$, complex variance-covariance matrices and finding an approximation for the distribution of this test statistic under the hypothesis of equality. If the conclusion of such an analysis is that the parameters in the underlying Wishart distributions are not constant, i.e. we have a non-stationary time series, then the question naturally arises, when do the changes actually occur? In this paper we present a factorization of the likelihood ratio

K. Conradsen and A. A. Nielsen are with DTU Compute, the Department of Applied Mathematics and Computer Science, H. Skriver is with DTU Space, the National Space Institute. Both departments are with the Technical University of Denmark, DK-2800 Kgs. Lyngby, Denmark. Correspondence to AAN, e-mail alan@dtu.dk, internet http://people.compute.dtu.dk/alan. 
statistic into a product of test statistics that each test simpler hypotheses of homogeneity up to a certain point and that are independent if the hypothesis of total homogeneity is true. We show how this may be used in setting up a change detector for solving the said problem.

The first results in this paper are direct generalizations of the $k=2$ case reported in [22] and further described in [28]. As mentioned above some other studies have been reported on change detection between two polarimetric SAR images, but the approach presented in this paper of detecting changes in a series of polarimetric SAR data in the covariance matrix representation is new. The launches of a number of satellite SAR systems during the latest more than 10 years have made it more common to use SAR data and especially time series of SAR data for different applications. The SAR systems include e.g. the ALOS satellites, the Radarsat satellites, the TerraSAR-X, the COSMOSkyMed satellites, and the Sentinel-1 mission. Some of these systems have a full polarimetric mode, whereas others have single and/or dual polarization. The time series of SAR data enable a number of applications where a core method for the utilization of the data is detection of changes in the time series, e.g. detection of a specific event for an agricultural crop (e.g. sowing or harvesting), detection of a flooding event in a monitoring system, or detection of changes in an urban area.

Section II includes a description of the covariance representation of polarimetric SAR data and describes the data set we have used in the demonstration of the test statistics. Section III gives the basic results on the omnibus test statistic and on its decomposition into a product of test statistics that test simpler hypotheses in the general, complex Wishart case. Since the real Gamma distribution is a univariate special case, the theory is exemplified on this distribution. Also, a scheme for using the test statistics in a change detection method to detect the changes in a time series is shown. Section IV shows the results of change detection in multi-temporal polarimetric data over five time points for the omnibus test and six time points for the decomposition, respectively. Section V contains the conclusions. An appendix gives details on the new test statistics and their distributions.

\section{DATA}

This section first describes the covariance representation of multilook polarimetric SAR data followed by a description of the SAR data used in the study.

\section{A. Polarimetric SAR}

A fully polarimetric SAR measures the 2 by 2 complex so-called scattering matrix at each resolution cell on the ground. The scattering matrix relates the incident and the scattered electric fields, [29]. If $S_{r t}$ denotes the complex scattering amplitude for receive and transmit polarization $(r, t \in\{h, v\}$ for horizontal and vertical polarization), then reciprocity, which normally applies to natural targets, gives $S_{h v}=S_{v h}$ (in the backscattering direction using the backscattering alignment convention) [29]. Assuming reciprocity, the scattering matrix is represented by the threecomponent complex target vector $s=\left[S_{h h} S_{h v} S_{v v}\right]^{T}$, where the superscript $T$ denotes the matrix transpose.

The inherent speckle in the SAR data can be reduced by spatial averaging at the expense of spatial resolution. In this so-called multi-look case (below $n$ is the number of looks) a more appropriate representation of the backscattered signal is the covariance matrix in which the average properties of a group of resolution cells can be expressed in a single matrix formed by the outer products of the averaged target vectors. The sample covariance matrix is defined as [29]

$$
\begin{aligned}
\langle\boldsymbol{C}\rangle_{f u l l} & =\left\langle\boldsymbol{s}(i) \boldsymbol{s}(i)^{H}\right\rangle \\
& =\left[\begin{array}{lll}
\left\langle S_{h h} S_{h h}^{*}\right\rangle & \left\langle S_{h h} S_{h v}^{*}\right\rangle & \left\langle S_{h h} S_{v v}^{*}\right\rangle \\
\left\langle S_{h v} S_{h h}^{*}\right\rangle & \left\langle S_{h v} S_{h v}^{*}\right\rangle & \left\langle S_{h v} S_{v v}^{*}\right\rangle \\
\left\langle S_{v v} S_{h h}^{*}\right\rangle & \left\langle S_{v v} S_{h v}^{*}\right\rangle & \left\langle S_{v v} S_{v v}^{*}\right\rangle
\end{array}\right]
\end{aligned}
$$

where $\langle\cdot\rangle$ denotes ensemble averaging, the * denotes complex conjugation, and the superscript $H$ denotes the complex conjugate transpose. Reciprocity results in a covariance matrix with rank 3. $n\langle C\rangle$ follows a complex Wishart distribution.

Spaceborne instruments often transmit only one polarization, say horizontal, and receive both polarizations giving rise to dual polarization data, e.g. $S_{h h}$ and $S_{h v}$. In this case we have the components $\left\langle S_{h h} S_{h h}^{*}\right\rangle,\left\langle S_{h h} S_{h v}^{*}\right\rangle$ and $\left\langle S_{h v} S_{h v}^{*}\right\rangle$ only. The resulting covariance matrix

$$
\langle C\rangle_{d u a l}=\left[\begin{array}{ll}
\left\langle S_{h h} S_{h h}^{*}\right\rangle & \left\langle S_{h h} S_{h v}^{*}\right\rangle \\
\left\langle S_{h v} S_{h h}^{*}\right\rangle & \left\langle S_{h v} S_{h v}^{*}\right\rangle
\end{array}\right]
$$

has rank 2. The availability of full-polarimetric data allows us to extract dual-polarimetric subsets. 


\section{B. Multitemporal EMISAR Data Set}

The SAR data used in the study have been acquired by the fully polarimetric Danish airborne SAR system, EMISAR, which operates at two frequencies, $C$ band $(5.3 \mathrm{GHz} / 5.7 \mathrm{~cm}$ wavelength) and L band $(1.25 \mathrm{GHz} / 24 \mathrm{~cm}$ wavelength) [30]. The nominal one-look spatial resolution is $2 \mathrm{~m}$ by $2 \mathrm{~m}$, the ground range swath is approximately $12 \mathrm{~km}$ and typical incidence angles range from $35^{\circ}$ to $60^{\circ}$. The processed data from this system are fully calibrated by using an advanced internal calibration system [31]. In 1998 L-band data were acquired over a Danish agricultural test site on 21 March, 17 April, 20 May, 16 June, 15 July, and 16 August.

All acquisitions have been co-registered by identifying ground control points in the images and using an interferometric DEM acquired by the EMISAR system [18]. Before the resampling, the original one-look scattering matrix data have been transformed to covariance matrix data, and these data have been averaged to reduce the speckle by a cosine-squared weighted 9 by 9 filter. The new pixel spacing in the images is $5 \mathrm{~m}$ by $5 \mathrm{~m}$, and the effective spatial resolution is approximately $8 \mathrm{~m}$ by $8 \mathrm{~m}$ at mid-range. After the averaging the equivalent number of looks is approximately 13.

Figure 1 row-wise shows RGB combinations of the diagonal elements of the full polarimetry covariance matrix at L-band for March, April, May (top row, left to right) and June, July, August (bottom row, left to right), respectively. $\left\langle S_{h v} S_{h v}^{*}\right\rangle$ (red) is stretched linearly between $-36 \mathrm{~dB}$ and $-6 \mathrm{~dB},\left\langle S_{h h} S_{h h}^{*}\right\rangle$ (green) between $-30 \mathrm{~dB}$ and $0 \mathrm{~dB}$ and $\left\langle S_{v v} S_{v v}^{*}\right\rangle$ (blue) between $-24 \mathrm{~dB}$ and $0 \mathrm{~dB}$. The darker areas in the March and April images are bare surfaces corresponding to spring crops, and the very bright areas in all images are forest areas, primarily coniferous forest. The development of the crops during the growing season is clearly seen in the series of images from March to August.

The changes we are looking for are changes in the average properties of the pixels/fields, i.e., the speckle patterns are not correlated. The images used are acquired with about one month interval, and the areas used are vegetated areas, forest and agricultural fields. The speckle patterns of such areas are very likely uncorrelated after one month. We are dealing with incoherent change detection, where it seems realistic to assume that the measurements on the same pixel taken a month apart in scenes with natural vegetation are temporally independent.

\section{THEORY}

In this section we first discuss the challenges of the multiple testing problem. We then give the test statistic for the equality of several complex Wishart distributed matrices and the associated probability measure. Following this, the test statistic is factorized into independent test statistics for partial hypotheses. These two results are used in setting up the pixelwise change detector and this is finally used in defining the fieldwise change detector.

\section{A. The Multiple Testing Problem}

In our setting we consider pixels from a series of images taken at time points $t_{1}<\cdots<t_{k}$ with distributions characterized by - often multivariate - parameters $\mu_{1}, \cdots, \mu_{k}$. We are concerned with detecting changes in those parameters, i.e. in assessing situations like

$$
\mu_{1}=\cdots=\mu_{i-1} \neq \mu_{i}=\cdots=\mu_{\ell-1} \neq \mu_{\ell}=\cdots=\mu_{k}
$$

which states that we have change only after time point $i-1$ and again after time point $\ell-1$. A simple approach would be sequentially testing hypotheses $\mu_{j}=\mu_{j-1}$ against $\mu_{j} \neq \mu_{j-1}$. However, this may give tests with a large false negative rate. For example, small gradual changes may not be detected, even when there is a clear trend throughout the time series. Therefore, we suggest to apply an omnibus test of hypothesis $H_{0}: \mu_{1}=\mu_{2}=\cdots=\mu_{k}$ against all alternatives, [27]. If this test is accepted, we conclude that no changes have occurred in the time interval [ $\left.t_{1}, t_{k}\right]$. If we reject the hypothesis, we may determine the occurrence of changes by suitable post hoc analysis using the results in Section III-C.

To clarify some concepts let us briefly summarize some definitions in hypothesis testing theory. In general, if we test a statistical hypothesis $H_{0}$, i.e. the item studied has no effect (here corresponding to no change over time), against the alternative $H_{1}$, i.e. the item has an effect (here corresponding to change over time), we may commit two different types of errors, i.e.

- Type I error: Rejecting a true hypothesis (false positive, false alarm). The significance level $\alpha$ of the test or the false positive rate is the probability of committing a type I error, or, if we have a composite hypothesis, the maximum of the possible probabilities.

- Type II error: Accepting a false hypothesis (false negative). The type II error rate, miss rate or the false negative rate $\beta$ is the probability of committing a type II error. The power of the test is $1-\beta$.

If a decision procedure is based on multiple (say $n$ ) independent tests, each with significance level $\alpha_{c}$, then the combined significance level $\alpha=\alpha_{F W E R}$, the so-called Family-Wise Error Rate is determined as $\alpha=\alpha_{F W E R}=1-\left[1-\alpha_{c}\right]^{n}$. This error rate increases with $n$. For $\alpha_{c}=0.05$ and $n=5,10$ and 15 we obtain the values 0.2262, 0,4013, and 0.5367, 

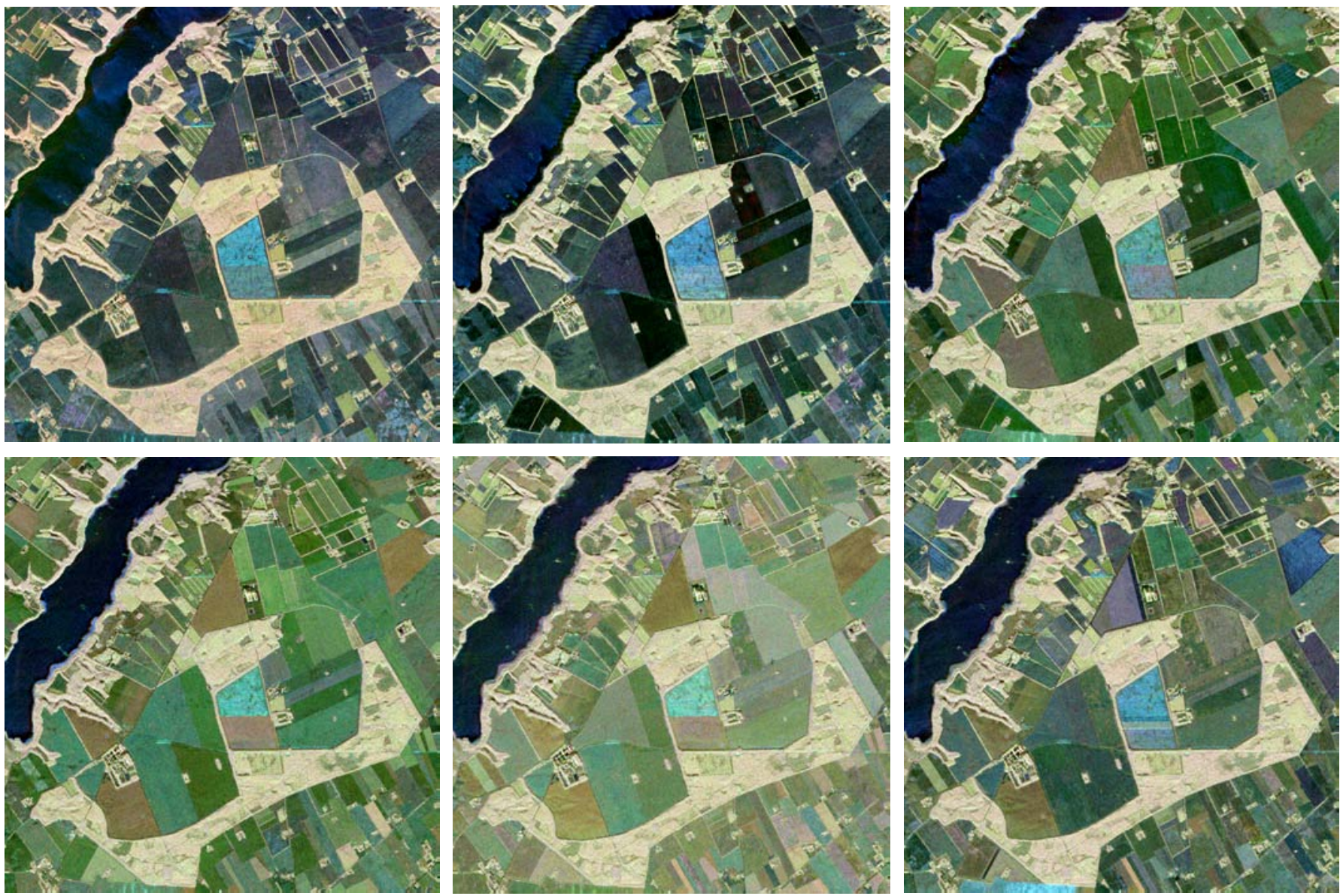

Figure 1. RGB images of diagonal elements of the L-band data March, April, May (top row, left to right), June, July, August (bottom row, left to right).

thus giving rather substantial false positive rates. If the individual tests are not independent, we can still say $\alpha=$ $\alpha_{F W E R} \leq n \alpha_{c}$. This is used in the so-called Bonferroni correction, where we put the per comparison significance level $\alpha=\alpha_{F W E R} / n$ in order to control the family-wise error, see for example [32].

Example. We shall briefly outline some of the problems of multiple comparisons by a very simple example. We consider independent random variables $X_{1}, \cdots, X_{k}$ with means $\mu_{1}, \cdots, \mu_{k}$ and (known) standard deviation $\sigma$. We furthermore consider two different types of changes corresponding to two simple mean value structures, namely $i)$, a signal which is linearly increasing with time (a constant increase of $2 \sigma$ from one time period to the next), or $i i$ ), a piecewise constant signal with a jump/step at a given time point (a constant value until time point $t_{j}$, where it jumps $s \sigma$ and again constant from then on), cf. Figure 2.

In situation $i$ ) it is difficult to detect the changes by comparing successive observations. If we are using standard statistical test using the test statistic $U_{m}=\left(X_{m}-X_{m-j+1}\right) /(\sqrt{2} \sigma)$ and significance level $5 \%$, the false negative rate is as high as $70.70 \%$ for $j=2$. However, if we consider comparisons between measurements corresponding to $j=3,4$ and 5 , the false negative rates will decrease to $19.26 \%, 1.12 \%$, and $0.01 \%$, but at the cost of an increased family-wise error rate, cf. the above.

If we try to compensate for this increased FWER by using the Bonferroni correction for 10 comparisons, i.e. use $\alpha=0.5 \%$ giving $\alpha_{F W E R} \leq 5 \%$, the false negative rates will be $91.82 \%, 49.15 \%, 7.56 \%$, and $0.22 \%$ for $j=2,3,4$, and 5 , i.e. considerably higher than above. Thus, it may be difficult to control the family-wise error rate and at the same time avoiding that the false negative rate is unnecessarily large.

If we instead use the omnibus test in the case with true means $\mu, \mu+2 \sigma, \mu+4 \sigma, \mu+6 \sigma, \mu+8 \sigma$ (situation $i$ ), the (likelihood ratio) test statistic is $\Sigma\left(X_{i}-\bar{X}\right)^{2} / \sigma^{2}$, which is non-centrally chi-squared distributed with $k-1$ degrees of freedom and non-centrality parameter $\Sigma\left(\mu_{i}-\bar{\mu}\right)^{2} / \sigma^{2}$. If we use significance level $5 \%$, the false negative rate becomes 


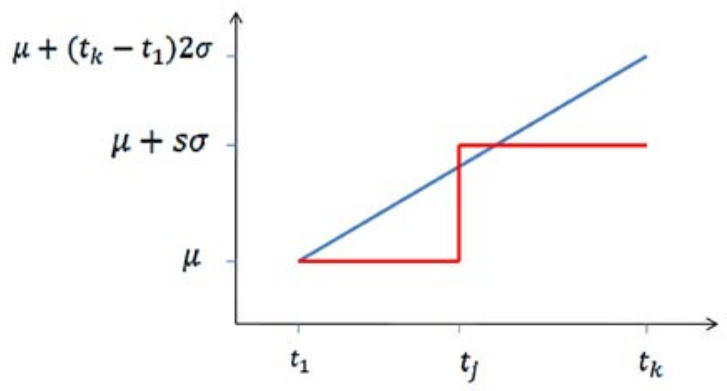

Figure 2. The two mean value situations $i$ and $i$ considered in the text. The blue line corresponds to a steady increase, the red to a discontinuous jump.

$0.02 \%$. If the same overall change in the means happens instantaneously after time period 3 (situation ii), the true means are $\mu, \mu, \mu, \mu+8 \sigma, \mu+8 \sigma$ (corresponding to situation ii)) and the false negative rate becomes $0.00 \%$. This example enhances advantages of the omnibus test with respect to limiting the error rates.

\section{B. Test for Equality of Several Complex Covariance Matrices}

As stated in Section II-A the sample covariance matrix of multilook, fully polarimetric SAR data when multiplied by the number of looks will follow a complex Wishart distribution. In order to test for possible changes between several, say $k$, time points, we therefore must investigate whether we may assume that several sample covariance matrices have the same expected value, say $\Sigma$, or whether we must assume that the expected values are different, equal to say $\Sigma_{i}, i=1, \ldots, k$, where at least two $\Sigma_{i}$ differ. In a general setting we therefore consider independent random variables $\boldsymbol{X}_{i}, i=1, \ldots, k$ that follow complex Wishart distributions

$$
\boldsymbol{X}_{i} \sim W_{C}\left(p, n, \Sigma_{i}\right), i=1, \ldots, k
$$

where $\mathrm{E}\left\{\boldsymbol{X}_{i} / n\right\}=\boldsymbol{\Sigma}_{i}$ and want to test the null hypothesis $H_{0}$

$$
H_{0}: \Sigma_{1}=\Sigma_{2}=\cdots=\Sigma_{k}
$$

against all alternatives, we use the following test statistic (see appendix for the derivation; for the real case see [33]; for the case with two complex matrices see [22])

$$
\begin{aligned}
Q & =k^{p n k} \frac{\prod_{i=1}^{k}\left|\boldsymbol{X}_{i}\right|^{n}}{|\boldsymbol{X}|^{n k}} \\
& =\left\{k^{p k} \frac{\prod_{i=1}^{k}\left|\boldsymbol{X}_{i}\right|}{|\boldsymbol{X}|^{k}}\right\}^{n} .
\end{aligned}
$$

Here $|\cdot|$ denotes the determinant, the independent $\boldsymbol{X}_{i}=n\langle\boldsymbol{C}\rangle_{i}$ follow the complex Wishart distribution, i.e., $\boldsymbol{X}_{i} \sim$ $W_{\mathbb{C}}\left(p, n, \Sigma_{i}\right)$, and $\boldsymbol{X}=\sum_{i=1}^{k} \boldsymbol{X}_{i} \sim W_{C}(p, n k, \boldsymbol{\Sigma})$, where $n$ is the number of looks. Also under $H_{0}, \hat{\boldsymbol{\Sigma}}=\boldsymbol{X} /(k n) . Q \in[0,1]$ with $Q=1$ for equality. For the logarithm of the test statistic we get

$$
\ln Q=n\left\{p k \ln k+\sum_{i=1}^{k} \ln \left|X_{i}\right|-k \ln |X|\right\} .
$$

If

$$
\begin{aligned}
f & =(k-1) p^{2} \\
\rho & =1-\frac{\left(2 p^{2}-1\right)}{6(k-1) p}\left(\frac{k}{n}-\frac{1}{n k}\right) \\
\omega_{2} & =\frac{p^{2}\left(p^{2}-1\right)}{24 \rho^{2}}\left(\frac{k}{n^{2}}-\frac{1}{(n k)^{2}}\right)-\frac{p^{2}(k-1)}{4}\left(1-\frac{1}{\rho}\right)^{2}
\end{aligned}
$$


then the probability of finding a smaller value of $-2 \rho \ln Q$ is

$$
\begin{aligned}
& P\{-2 \rho \ln Q \leq z\} \simeq P\left\{\chi^{2}(f) \leq z\right\} \\
& \quad+\omega_{2}\left[P\left\{\chi^{2}(f+4) \leq z\right\}-P\left\{\chi^{2}(f) \leq z\right\}\right],
\end{aligned}
$$

where $z=-2 \rho \ln q$ and $q$ is a particular realization (an observed value) of the stochastic variable $Q$. Instead of $q$ we may write $q_{\mathrm{obs}} ; q$ and $q_{\mathrm{obs}}$ are used interchangeably below. See also the appendix.

For full polarimetry data $p=3$, for dual polarimetry $p=2$, and for single band (HH, HV or VV) data $p=1$. In the latter case $X_{i}$ and $X$ are Gamma distributed scalar random variables $X_{i}$ and $X$, and $Q$ becomes

$$
Q=\left\{k^{k} \frac{\prod_{i=1}^{k} X_{i}}{X^{k}}\right\}^{n} .
$$

For two time points, i.e., $k=2$ this is equivalent to the classical ratio detector [1], [2].

\section{Test for Equality of First $j<k$ Complex Covariance Matrices}

If the above test shows that we cannot reject the hypothesis of equality, no change has occurred over the time span covered by the data. If we, on the other hand, can reject the hypothesis, change has occurred at some time point. In order to establish at which time a change has occurred we shall use the fact that the likelihood ratio test statistic may be decomposed into a product of test statistics that test simpler hypotheses and that are independent if $H_{0}$ is true. To test whether the first $j, 1<j<k$ complex covariance matrices $\Sigma_{i}(p$ by $p$ ) are equal, i.e., given that

$$
\Sigma_{1}=\Sigma_{2}=\cdots=\Sigma_{j-1}
$$

then the likelihood ratio test statistic $R_{j}$ for testing the hypothesis

$$
H_{0, j}: \Sigma_{j}=\Sigma_{j-1} \text { against } H_{1, j}: \Sigma_{j} \neq \Sigma_{j-1}
$$

is

$$
\begin{aligned}
R_{j} & =\frac{j^{j p n}}{(j-1)^{(j-1) p n}} \frac{\left|\boldsymbol{X}_{1}+\cdots+\boldsymbol{X}_{j-1}\right|^{(j-1) n}\left|\boldsymbol{X}_{j}\right|^{n}}{\mid \boldsymbol{X}_{1}+\cdots+\boldsymbol{X}_{j}^{j n}} \\
& =\left\{\frac{j^{j p}}{(j-1)^{(j-1) p}} \frac{\left|\boldsymbol{X}_{1}+\cdots+\boldsymbol{X}_{j-1}\right|^{(j-1)}\left|\boldsymbol{X}_{j}\right|}{\left|\boldsymbol{X}_{1}+\cdots+\boldsymbol{X}_{j}\right|^{j}}\right\}^{n}
\end{aligned}
$$

or

$$
\begin{aligned}
\ln R_{j}= & n\{p(j \ln j-(j-1) \ln (j-1)) \\
& \left.+(j-1) \ln \left|\sum_{i=1}^{j-1} \boldsymbol{X}_{i}\right|+\ln \left|\boldsymbol{X}_{j}\right|-j \ln \left|\sum_{i=1}^{j} \boldsymbol{X}_{i}\right|\right\}
\end{aligned}
$$

Furthermore

$$
Q=\prod_{j=2}^{k} R_{j},
$$

and if $H_{0}$ is true, then the random variables $R_{2}, \cdots, R_{k}$ are independent.

Finally, letting

$$
\begin{aligned}
f & =p^{2} \\
\rho_{j} & =1-\frac{2 p^{2}-1}{6 p n}\left(1+\frac{1}{j(j-1)}\right) \\
\omega_{2 j} & =-\frac{p^{2}}{4}\left(1-\frac{1}{\rho_{j}}\right)^{2}+\frac{1}{24 n^{2}} p^{2}\left(p^{2}-1\right)\left(1+\frac{2 j-1}{j^{2}(j-1)^{2}}\right) \frac{1}{\rho_{j}^{2}}
\end{aligned}
$$

we get

$$
\begin{aligned}
& P\left\{-2 \rho_{j} \ln R_{j} \leq z\right\} \simeq P\left\{\chi^{2}(f) \leq z\right\} \\
& +\omega_{2 j}\left[P\left\{\chi^{2}(f+4) \leq z\right\}-P\left\{\chi^{2}(f) \leq z\right\}\right]
\end{aligned}
$$


where $z=-2 \rho_{j} \ln r_{j}$ and $r_{j}$ is a particular realization (an observed value) of the stochastic variable $R_{j}$. Instead of $r_{j}$ we may write $r_{j, \text { obs }} ; r_{j}$ and $r_{j, \text { obs }}$ are used interchangeably below. See the appendix which also gives the resulting formulas for the Gamma distributed scalar case.

\section{Pixelwise Change Detection}

We start by looking at the Gamma distributed case, cf. the appendix. This will allow a more intuitive presentation e.g. by plotting the power of the SAR signal as a function of time. Furthermore the notation becomes somewhat simpler. We have time points $t_{1}, \ldots, t_{k}$ corresponding to parameters $\beta_{1}, \ldots, \beta_{k}$ and introduce the global hypotheses

$$
H_{0}^{(\ell)}: \beta_{\ell}=\beta_{\ell+1}=\cdots=\beta_{k}, \ell=1, \ldots, k-1,
$$

i.e. the last $k-\ell+1$ of all the parameters are equal. Furthermore we consider the marginal hypotheses

$$
H_{0, j}^{(\ell)}: \beta_{\ell+j-1}=\beta_{\ell+j-2}\left(=\beta_{\ell+j-3}=\cdots=\beta_{\ell}\right), j=2, \ldots, k-\ell+1,
$$

i.e. the first $j$ of the parameters in the global hypothesis $H_{0}^{(\ell)}$ are equal.

The omnibus test statistic for testing $H_{0}^{(\ell)}$ against all alternatives based on $X_{\ell}, X_{\ell+1}, \ldots, X_{k}$ is

$$
Q^{(\ell)}=\left\{(k-\ell+1)^{k-\ell+1} \frac{X_{\ell} \cdots X_{k}}{\left(X_{\ell}+\cdots+X_{k}\right)^{k-\ell+1}}\right\}^{n} .
$$

Let us assume that $H_{0, j-1}^{(\ell)}$ is true, i.e. that the first $j-1$ parameters are equal or $\beta_{\ell}=\beta_{\ell+1}=\cdots=\beta_{\ell+j-2}$. Then the test statistic for testing $H_{0, j}^{(\ell)}$ against the alternative $\beta_{\ell+j-1} \neq \beta_{\ell+j-2}$, i.e. for testing that the $j$ th parameter is equal to the $j-1$ preceding ones becomes

$$
R_{j}^{(\ell)}=\left\{\frac{j^{j}}{(j-1)^{j-1}} \frac{\left(X_{\ell}+\cdots+X_{\ell+j-2}\right)^{j-1} X_{\ell+j-1}}{\left(X_{\ell}+\cdots+X_{\ell+j-1}\right)^{j}}\right\}^{n}, j=2, \ldots, k-\ell+1
$$

and

$$
Q^{(\ell)}=R_{2}^{(\ell)} \cdots R_{k-\ell+1}^{(\ell)}
$$

with independence if $H_{0}^{(\ell)}$ is true.

In broad terms the algorithm becomes

1) Set $\ell=1$.

2) Test $H_{0}^{(\ell)}$ against all alternatives.

If accepted, conclude that there are no changes in the interval $\left[t_{\ell}, t_{k}\right]$. Go to 5 .

If rejected, conclude that there is at least one change in the interval $\left[t_{\ell}, t_{k}\right]$ and go to 3 .

3) Test the marginal hypotheses $H_{0, j}^{(\ell)}$ and let the first significant hypothesis be $H_{0, r+1}^{(\ell)}$.

Conclude that we have a change in $\left[t_{\ell+r-1}, t_{\ell+r}\right]$.

4) Set $\ell=\ell+r$ and go to 2 .

5) Finish.

The algorithm is illustrated in the next example.

Example. We now consider (hypothetical), for example $\left\langle S_{h h} S_{h h}^{*}\right\rangle$ values (corresponding to 13-look EMISAR data) from eight time points. The observations are given in Table I. It is assumed that they represent independent realizations of Gamma distributed random variables $X_{i} \sim G\left(13, \beta_{i}\right), i=1, \ldots, 8$.

Table I. The (hypothetical) data CONSIDERED For the GAMma Distribution EXAMPLe.

\begin{tabular}{|c|c|c|c|c|c|c|c|c|}
\hline$j$ & 1 & 2 & 3 & 4 & 5 & 6 & 7 & 8 \\
\hline$x_{j}$ & 1.3338 & 2.0683 & 1.3494 & 1.3858 & 0.0806 & 1.6302 & 1.5201 & 1.9932 \\
\hline
\end{tabular}

By direct computation we get that $-2 \ln Q=54.2510$, and comparing this to quantiles in a $\chi^{2}(7)$-distribution $((k-$ 1) $p^{2}=7$ for $k=8$ and $p=1$ ) shows that $P\left\{Q^{(1)}<q^{(1)}\right\} \simeq 0$, i.e., this value is significant at all reasonable levels. Therefore, we conclude that we have (at least) one change in the time period considered, i.e., $i=1, \ldots, 8$. We want to determine the time point for the first change. Therefore we successively compute the quantities $R_{j}^{(1)}$ and $P\left\{R_{j}^{(1)}<r_{j}^{(1)}\right\}, j=2, \ldots, 8$, 


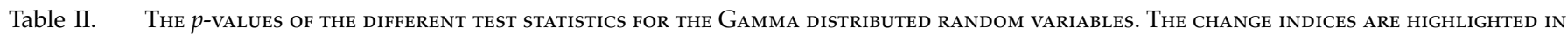
YELLOW AND THE $p$-VALUES FOR THE ASSOCIATED GLOBAL TESTS IN GREEN. WE NEED CALCULATE THE HIGHLIGHTED NUMBERS ONLY, THE OTHER NUMBERS ARE SHOWN FOR ILLUSTRATIVE PURPOSES.

\begin{tabular}{|c|c|c|c|c|c|c|c|}
\hline Marg. Hyp. & \multicolumn{7}{|c|}{ Global Hypotheses $H_{0}^{(\ell)}, \ell=1, \ldots, 7$} \\
\hline & $\beta_{1}=\cdots=\beta_{8}$ & $\beta_{2}=\cdots=\beta_{8}$ & $\beta_{3}=\cdots=\beta_{8}$ & $\beta_{4}=\cdots=\beta_{8}$ & $\beta_{5}=\cdots=\beta_{8}$ & $\beta_{6}=\cdots=\beta_{8}$ & $\beta_{7}=\beta_{8}$ \\
\cline { 2 - 8 }$H_{0, j}^{(\ell)}$ & $P\left\{R_{j}^{(1)}<r_{j}^{(1)}\right\}$ & $P\left\{R_{j}^{(2)}<r_{j}^{(2)}\right\}$ & $P\left\{R_{j}^{(3)}<r_{j}^{(3)}\right\}$ & $P\left\{R_{j}^{(4)}<r_{j}^{(4)}\right\}$ & $P\left\{R_{j}^{(5)}<r_{j}^{(5)}\right\}$ & $P\left\{R_{j}^{(6)}<r_{j}^{(6)}\right\}$ & $P\left\{R_{j}^{(7)}<r_{j}^{(7)}\right\}$ \\
\hline$\beta_{2}=\beta_{1}$ & 0.2653 & & & & & \\
$\beta_{3}=\beta_{2}$ & 0.5013 & 0.2780 & & & & \\
$\beta_{4}=\beta_{3}$ & 0.6801 & 0.5423 & 0.9459 & & & \\
$\beta_{5}=\beta_{4}$ & 0.0000 & 0.0000 & 0.0000 & 0.0000 & & & \\
$\beta_{6}=\beta_{5}$ & 0.3587 & 0.3378 & 0.0723 & 0.0151 & 0.0000 & & \\
$\beta_{7}=\beta_{6}$ & 0.6096 & 0.6057 & 0.2980 & 0.2129 & 0.0824 & & \\
$\beta_{8}=\beta_{7}$ & 0.1581 & 0.1642 & 0.0744 & 0.0636 & 0.0442 & 0.4853 & \\
\hline$P\left\{Q^{(\ell)}<q^{(\ell)}\right\}$ & 0.0000 & 0.0000 & 0.0000 & 0.0000 & 0.0000 & 0.7696 & 0.4903 \\
\hline
\end{tabular}

where the upper index (1) indicates that we are looking at the first global hypothesis presented in Table II, column 2 .

In the decomposition of the likelihood ratio test statistic $Q$ we see that $R_{5}^{(1)}$ is the first significant component corresponding to rejection of the hypothesis $\beta_{5}=\beta_{4}$. The conclusion is thus so far that we may assume that $\beta_{1}=$ $\beta_{2}=\beta_{3}=\beta_{4}$, and we must investigate whether there are changes in the period $i=5, \ldots, 8$, i.e., test the global hypothesis $H_{0}^{(5)}: \beta_{5}=\ldots=\beta_{8}$. Based on the observations $X_{5}, \ldots, X_{8}$ we compute the likelihood ratio test statistic for $H_{0}^{(5)}$ and find that $P\left\{Q^{(5)}<q^{(5)}\right\} \simeq 0$. We conclude that there is at least one change in the period considered and decompose the likelihood ratio statistic. We see that $R_{2}^{(5)}$ is the first significant component corresponding to rejection of the hypothesis $\beta_{6}=\beta_{5}$. We thus have a change between time points 5 and 6 . Then we must investigate whether there is a change between the last three time points, i.e., we consider the global hypothesis $H_{0}^{(6)}: \beta_{6}=\beta_{7}=\beta_{8}$. We find that $P\left\{Q^{(6)}<q^{(6)}\right\}=0.7696$, i.e., we assume that we have no changes in this period.

The conclusion is thus that we observe significant changes between

- $\beta_{4}$ and $\beta_{5}$,

- $\beta_{5}$ and $\beta_{6}$,

and therefore we conclude that we have the following distinct populations

- $\beta_{1}=\beta_{2}=\beta_{3}=\beta_{4}$,

- $\beta_{5}$,

- $\beta_{6}=\beta_{7}=\beta_{8}$.

Remark. If we introduce the term change index for the relevant quantities $P\left\{R_{j}^{(\ell)}<r_{j}^{(\ell)}\right\}$, we have that large values of one minus the change index correspond to changes. From a statistical point of view, the threshold 0.95 seems natural. In the Gamma case we may plot the observations as well as the quantities one minus the change index in the same coordinate system. This is done in Figure 3 which illustrates the outcome of the change detection algorithm.

The above description of the change detection algorithm may immediately be generalized to the complex Wishart distribution by simply replacing the parameter $\beta$ with $\Sigma$ and by using (6) and (15) in setting up the global and marginal test statistics based on the last $k-\ell+1$ observations. A detailed description of the algorithm is given in Figure 4.

\section{E. Fieldwise Change Detection}

Before defining the change index for a field, let us initially state some facts from statistical testing theory. The $p$-value of a statistical test is the probability of getting a test statistic that is at least as extreme as the one observed, assuming that the null hypothesis is actually true (and that the assumptions of the analysis are met). If the $p$-value is smaller than the prescribed significance level $\alpha$ (e.g. $\alpha=0.05$ ) we reject the hypothesis since the discrepancy between the data and the hypothesis is too large.

Furthermore, if the sampling distribution is continuous, the distribution of the $p$-values will be uniform over the interval $[0,1]$ if the null hypothesis is true. This implies, of course, that if the same experiment is replicated many times, if the hypothesis is true and if we test on a $5 \%$ level of significance, then $5 \%$ of the $p$-values will fall in the interval [0,0.05], and the remaining $p$-values will be larger than 0.05. If the null hypothesis is not true, then the 


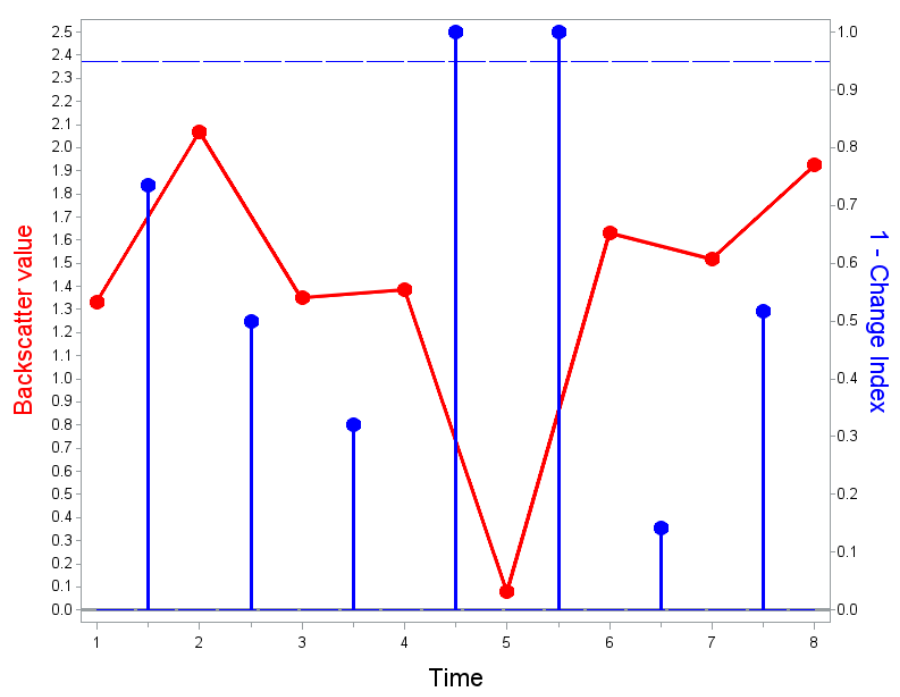

Figure 3. The (hypothetical) observations and the change indices for the Gamma distribution example.

fraction of $p$-values falling in the interval $[0,0.05]$ will in general be much larger than $5 \%$. How large, depends on the power of test.

The change index for a field may now be defined by first considering the empirical distributions of the different $p$-values obtained (i.e., the $P\{Q<q\}=1-P\{-2 \rho \ln Q \leq-2 \rho \ln q\}$ and $P\left\{R_{j}^{(\ell)}<r_{j}^{(\ell)}\right\}=1-P\left\{-2 \rho \ln R_{j}^{(\ell)} \leq-2 \rho \ln r_{j}^{(\ell)}\right\}$ values) for each pixel observed over the time span considered and then apply the one-pixel definition on suitable measures of location for those distributions, like for example mean or median.

\section{Results}

In this section the data set described in Section II-B is used to illustrate different aspects of the test statistics, and in that process we utilize different parts of the time series to provide illustrative examples. We first show the strength of the omnibus test statistic $Q$ when applied to three different areas covering forest, a rye field, and a grass field, respectively, using data over five time points from March to July only. We then proceed to show the power of the factorization of $Q$ into the $R_{j} \mathrm{~s}$, this time using data from six time points from March to August.

\section{A. Change Indices for Three Different Cases: Forest, Rye and Grass}

Figure 5 shows $-2 \rho \ln q_{\text {obs }}$ for full polarimetry (left image), and $P\left\{Q \geq q_{\mathrm{obs}}\right\}$ (right image). Here $\rho=0.91282$ and $\omega_{2}=0.023577$. The left-hand image shows low values for the forest areas, which indicates that no changes have occurred during the five time points, which is a reasonable results for the coniferous forest areas.

In order to provide explanations of the results for the test statistics the following analysis will be based on results from areas like fields with a given crop, where we have in situ information on ground usage. In this subsection we show the results for three cases with different change patterns over time: a forest area with no changes, a rye field where changes may be detected by both the omnibus test and the pairwise tests, and a grass area where changes may be detected only by the omnibus test and not the pairwise tests.

Backscatter coefficients for these areas are shown in Figure 6, and polarimetric entropy and alpha angle parameters from the Cloude-Pottier eigenanalysis decomposition [34] are shown in Figure 7. The forest area has a very constant backscatter through all the images. The entropy for the forest area is very close to one for all acquisitions, and the alpha angle is approximately $50^{\circ}$, which indicates as expected that the backscatter from the forest is dominated by volume scattering. It is clearly seen from these results, that no changes occur for the forest area through all the acquisitions.

For the grass and rye areas, on the other hand, the backscatter coefficients as well as the entropy and alpha angle parameters change through the time series. For the rye field the entropy and alpha angles values show for the first four acquisitions that the backscattering mechanism is the medium entropy surface scattering type [34], which indicates rough surface scattering with canopy penetration effects. Rye is a relatively sparse crop, which corresponds to the results that the backscatter is dominated by surface scattering affected by the vegetation layer. Between April and May we see an increased influence of the vegetation layer by the increase in the HH backscatter (cf. Figure 6(b)). 


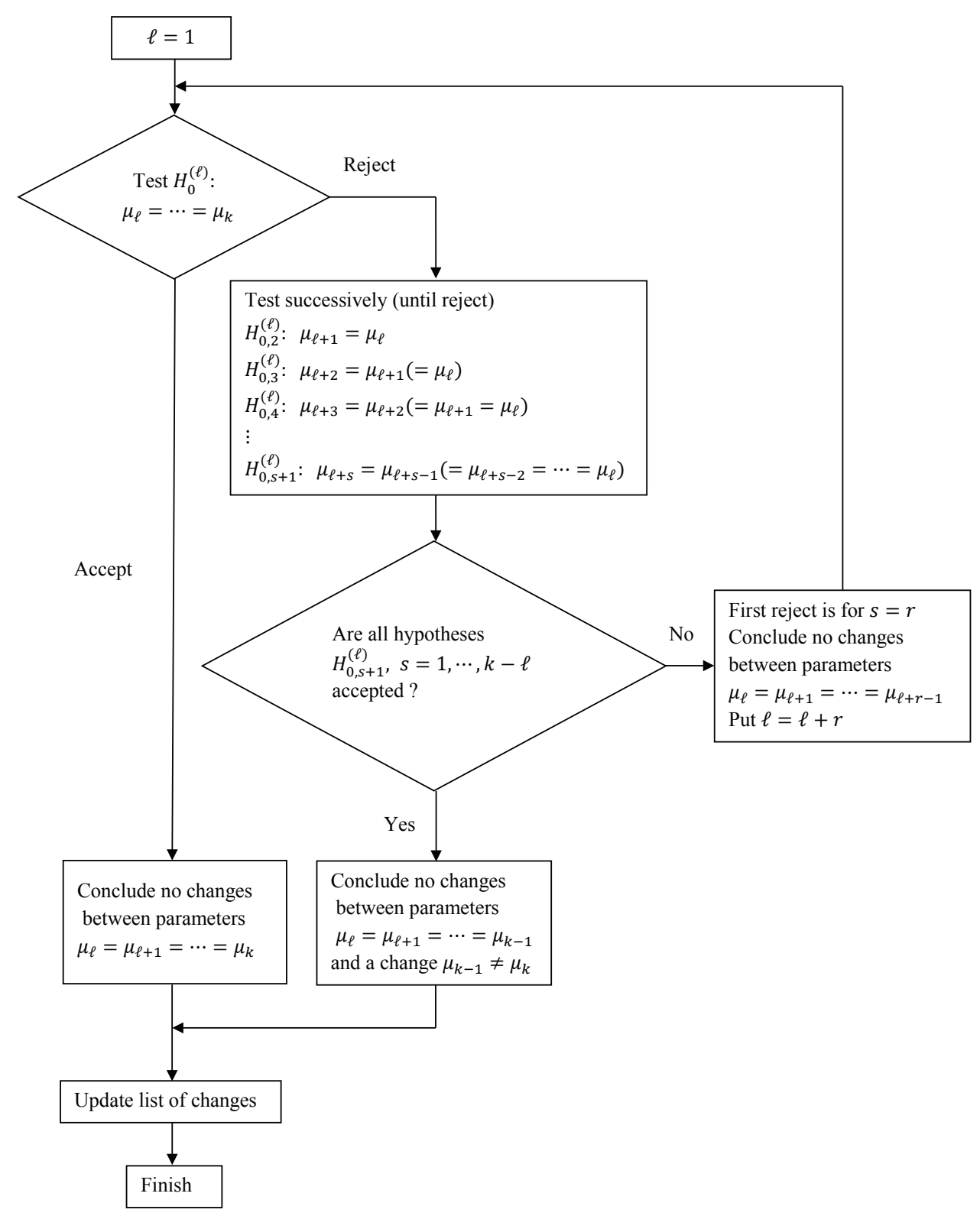

Figure 4. Flow chart for the change detection method. $\mu$ is a generic name for the unknown parameters considered. In the leftmost part we are investigating whether there are changes in the interval $\left[t_{l}, t_{k}\right]$ using the $Q$-test statistic (6). If the answer is no, the analysis is finished. If the answer is yes, i.e. we have at least one change in $\left[t_{l}, t_{k}\right]$, we go to the column in the middle. Based on observations at $t_{\ell}, \cdots, t_{\ell+s}$ we (for $s=1, \cdots, k-1$ ) successively investigate whether there are changes between time points $t_{\ell+s-1}$ and $t_{\ell+s}$. This is done by testing the hypothesis $H_{0, j+1}^{\ell}: \mu_{\ell+j}=\mu_{\ell+j-1}$ corresponding to $H_{0, j}$ from (14) using the test statistic $R_{j}^{(\ell)}$ corresponding to $R_{j}$ from (15). If we do not identify any changes before time point $t_{k-1}$ we conclude that the change in the interval $\left[t_{\ell}, t_{k}\right]$ falls in the interval $\left[t_{k-1}, t_{k}\right]$. If the first change we identify, occurs in $\left[t_{\ell+r-1}, t_{\ell+r}\right]$ we conclude that there are no changes in the interval $\left[t_{l}, t_{\ell+r-1}\right]$ and a change in $\left[t_{\ell+r-1}, t_{\ell+r}\right]$. We then update $\ell$ to $\ell+r$ and start again in the leftmost column. 


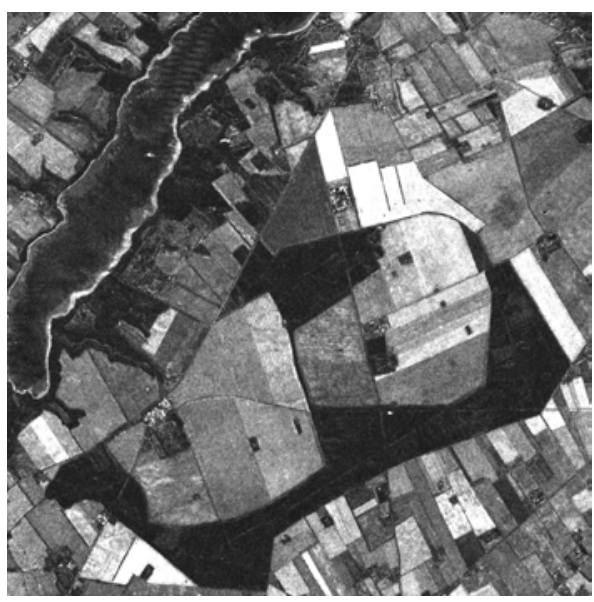

(a)

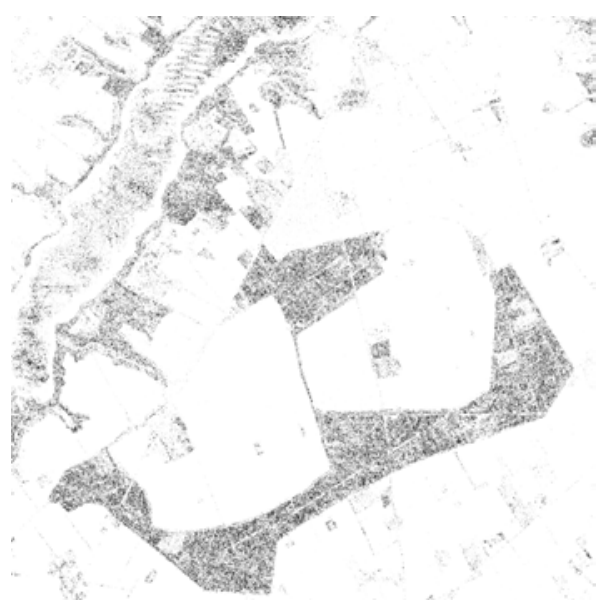

(b)

Figure 5. (a) $-2 \rho \ln q_{\mathrm{obs}}$ for full polarimetry (stretched linearly between 0 and 300), and (b) $P\left\{Q \geq q_{\mathrm{obs}}\right\}$, both based on data from March to July. In both images low values (dark tones) correspond to no change.

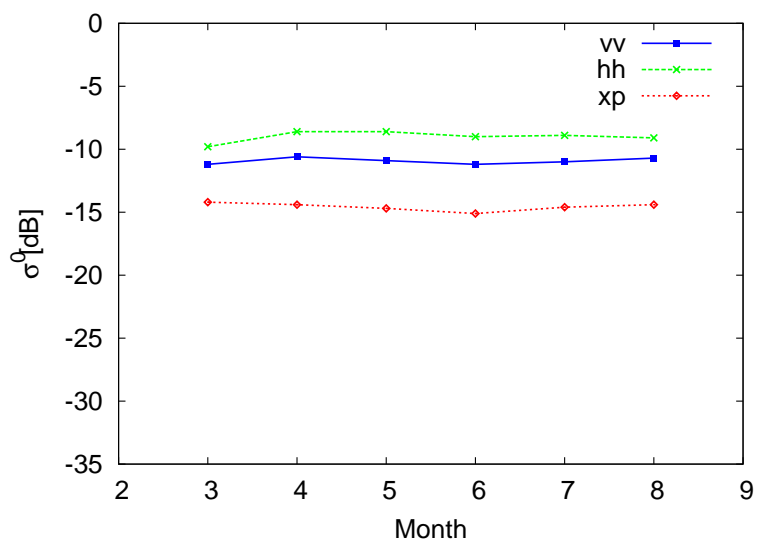

(a)

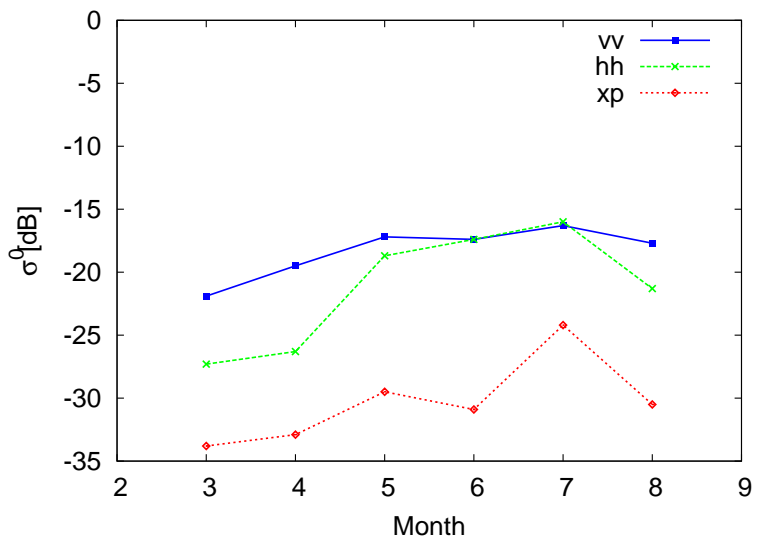

(b)

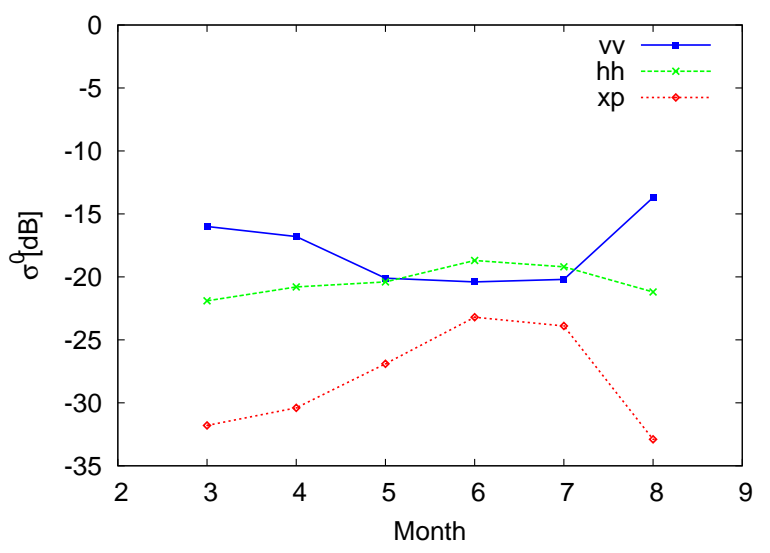

(c)

Figure 6. Backscatter coefficients for forest (a), rye (b), and grass (c). In the legend vv denotes $\left\langle S_{v v} S_{v v}^{*}\right\rangle$, hh denotes $\left\langle S_{h h} S_{h h}^{*}\right\rangle$, and $\mathrm{xp}$ denotes $\left\langle S_{h h} S_{v v}^{*}\right\rangle$. 


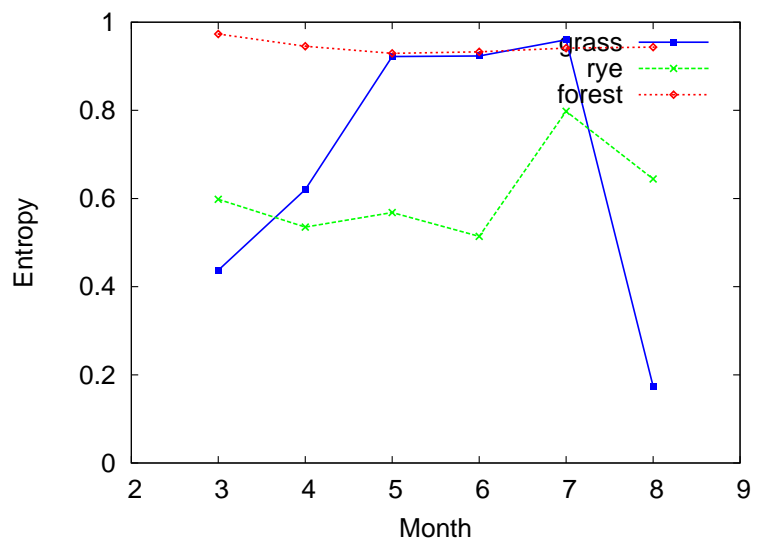

(a)

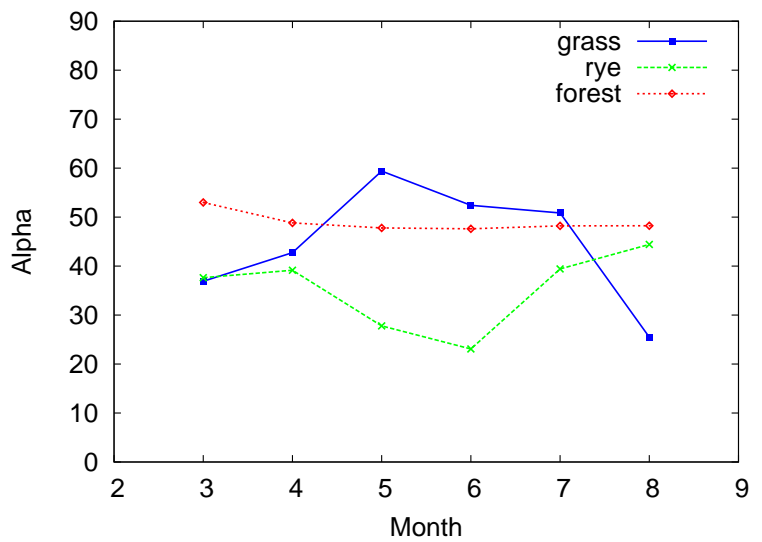

(b)

Figure 7. Entropy (a) and alpha angle (b) for grass, rye, and forest. Entropy has no unit, alpha angle is in degrees.

Table III. The AVERAgE PROBAbILITIES OF OBTAINING MORE EXTREME VALUES OF THE TEST STATISTIC FOR THE FOREST, RYE AND GRASS AREAS FOR PAIRWISE COMPARISONS AND FOR THE OMNIBUS TEST, DATA FROM MARCH TO July. VAlueS belOW 0.05 INDiCATE STATISTICALly SignifiCANT CHANGES.

\begin{tabular}{|c|c|c|c|c|c|}
\hline Average $P\left\{Q<q_{o b s}\right\}$ & \multicolumn{3}{|c|}{ Pairwise comparisons } & Omnibus test \\
\hline Hypothesis $H_{0}^{(\ell)}$ & Mar=Apr & Apr=May & May=Jun & Jun=Jul & Mar=Apr=May=Jun=Jul \\
\hline Forest & 0.3925 & 0.6235 & 0.4913 & 0.4567 & 0.3823 \\
Rye & 0.4249 & 0.0131 & 0.3344 & 0.0242 & 0.0000 \\
Grass & 0.2808 & 0.0638 & 0.1244 & 0.4311 & 0.0001 \\
\hline
\end{tabular}

For the July acquisition the vegetation layer has become so dense that we now see volume scattering, i.e., increased cross-polarized backscatter (cf. Figure 6(b)), and increased entropy (cf. Figure 7(a)). Finally, for the August acquisition the crop is now dried out, so less volume scattering occurs, and hence decreasing cross-polarized backscatter and smaller entropy.

The scattering mechanism for the grass area changes from surface scattering affected by the vegetation layer in March and April with entropy and alpha angles values similar to the rye field (cf. Figure 7), over volume scattering in May with some contribution of double-bounce scattering with an alpha angle of about $60^{\circ}$ (cf. Figure $7(\mathrm{~b})$ ), to full volume scattering in June and July with the entropy close to 1 (cf. Figure 7(a)). These changes correspond to the growth and conditions of the grass. In March, April and May the radar wave can penetrate the not so dense grass vegetation, and in May the vegetation is, however, sufficiently dense to result in volume scattering from the vegetation. At the same time the vegetation is still so sparse that some double bounce scattering can occur, and in the June and July acquisitions the vegetation is so dense, that volume scattering occurs. In August, the grass is cut, and hence the entropy and alpha angle now show surface scattering behavior (cf. Figure 7), and the VV-backscatter increases and the cross-polarized backscatter decreases (cf. Figure 6(c)).

Table III shows the average probabilities of the test statistics for the forest, the rye and the grass areas using the first five acquisitions. The values shown are the average values over all pixels for each area of the observed significance levels $\alpha_{o b s}$ (i.e. the probabilities $P\left\{Q<q_{o b s}\right\}$ of getting values of the test statistic that are more extreme than the observed under the null hypothesis). In the table both the results of performing consecutive pairwise comparisons between the acquisitions (corresponding to the test statistic in [22]), and the results of using the omnibus test statistic presented in this paper (cf. (11)).

It is clear that no change can be detected for the forest area, neither in the pairwise comparisons nor in the omnibus test. For the rye area the omnibus test clearly detects change in the time series. From the pairwise comparisons, it is seen, that changes can be detected at a 5\% significance level between the April and May acquisitions, and between the June and July acquisitions, corresponding to the observed changes in the polarimetric parameters described above.

The omnibus test for the grass area shows clearly that the parameters over the five acquisitions have not been constant, i.e. changes have occurred during this period. The pairwise comparisons, however, show no changes at a 5\% significance level. Even though the polarimetric analysis above describes some relatively significant changes during the five acquisitions, the pairwise comparisons do not pick up these changes. This result shows the power of the omnibus test, where the changes between the consecutive images are too small to be detected, but the overall change during all the acquisitions is significant. 


\section{B. The Points of Change for Three Different Cases: Forest, Rye and Grass}

In Table IV we have shown the $p$-values for the forest area, and the corresponding path leading to the change index for the forest area computed by looking at the averages described above. In Table $\mathrm{V}$ we have shown the equivalent results for a rye field, and in Table VI for a grass field.

In Tables IV-VI the first number in each column corresponds to tests for the relevant pairwise comparisons, see (6) where $k=2$ in the pairwise case.

For the forest results in Table IV is it seen from the $P\{Q<q\}$ value in the first column that no change has occurred for all acquisitions (with a significance level of 0.05). This is also seen from the individual $P\left\{R_{j}^{(\text {Mar })}<r_{j}^{(\text {Mar })}\right\}$ values in the first column.

For the rye results in Table $\mathrm{V}$ the $P\{Q<q\}$ value for the first column shows that a change has occurred, and according to the $P\left\{R_{j}^{(\text {Mar })}<r_{j}^{(\text {Mar })}\right\}$ values in the first column this change has occurred between April and May. Now moving to the third column in Table $\mathrm{V}$, the $P\{Q<q\}$ value here shows that a change has occurred between May and August. The $P\left\{R_{j}^{(\text {May })}<r_{j}^{(\text {May })}\right\}$ values in the third column shows that the change has occurred between June and July. Finally, moving to the fifth column, it is seen that a change has occurred between July and August. We thus conclude that we have the following distinct populations for rye

1) March = April,

2) May = June,

3) July, and

4) August.

Using the same methodology for grass in Table VI we find that changes have occurred between April and May, and between July and August. In this case the distinct populations are

1) March = April,

2) May = June $=$ July, and

3) August.

These populations are consistent with the analysis of the polarimetric parameters described in Section IV-A. The forest areas clearly show no change for all six acquisitions. The rye field has four distinct populations, i.e., MarchApril, May-June, July, and August, as seen above. For March and April, surface scattering is dominating, and in May and June an increased contribution from volume scattering is seen. In July, more volume scattering is seen, and finally, in August less volume scattering is seen, due to drying out of the crop. For the grass field, three distinct populations are identified, i.e. March-April, May-June-July, and August. From the backscatter coefficients in Figure 6(c), and the entropy and alpha angle in Figure 7 groupings of the parameters according to these populations are clearly seen, and it also corresponds to the analysis of the scattering mechanisms given in Section IV-A.

\section{Distribution of the $p$-Values for Forest and Grass}

The question naturally arises whether the chosen mean values are proper descriptors of the underlying distributions.

In Figure 8 the histograms (presented rowwise) behind the six $p$-values that constitute the change index for forest are shown. For the forested area no changes are seen, and the means of the $p$-values $(\bar{p})$ for the omnibus test $P\{Q<q\}(\bar{p}=$ $0.3494)$ and for the marginal tests $P\left\{R_{j}^{(\ell)}<r_{j}^{(\ell)}\right\}(\bar{p}=0.3925,0.5469,0.4121,0.4229$, and 0.4116) are fairly representative for the histograms. We only have minor deviations between the histograms and the theoretical uniform distribution that we would get assuming that no changes have occurred. The reason for these minor deviations is probably twofold: Firstly there may very well be changes in small patches, and secondly, some of the assumptions behind the sampling theory (independence of observations, distributional properties) may not be fulfilled.

The grass field shows a slightly more complicated scenario. The first three histograms in Figure 9 show the distribution of the $p$-values for testing the hypotheses: total homogeneity (rejected, $\bar{p}=0.0000$ ), Apr $=$ Mar (accepted, $\bar{p}=0.2808)$, and May $=\operatorname{Apr}(=$ Mar) (rejected, $\bar{p}=0.0112)$. Having a change between May and Apr, we perform an omnibus test on the remaining months, i.e. we test May $=\mathrm{Jun}=\mathrm{Jul}=$ Aug. Here $\bar{p}=0.0000$ (histogram not shown), we reject and we test Jun = May (accept, $\bar{p}=0.1244)$, Jul $=$ Jun (= May) (accept, $\bar{p}=0.3879)$, and Aug $=\mathrm{Jul}(=\mathrm{Jun}=$ May) (reject, $\bar{p}=0.0000$ ). For the three $p$-values that are smaller than 0.05 it is seen from the histograms that almost all values (actually between $97.8 \%$ and $100 \%$ ) lie in the interval [0, 0.05] so a small mean $p$-value is consistent with all pixels in the area showing changes. For the values above 0.05 it follows that a substantial fraction of the observed $p$-values are larger than 0.05 , i.e., the majority of the corresponding pixels show no changes. However, e.g., in the case June = May (second row, second column) we have an over-representation of low $p$-values when comparing with the uniform distribution. This might be due to changes in smaller patches, possibly a sign of local delays of the changes we saw between May and Apr.

Thus, in both cases there is a good agreement between the conclusion based on the averages and what the distribution of the $p$-values indicates for the area considered. 


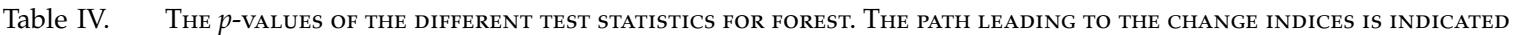

\begin{tabular}{|c|c|c|c|c|c|}
\hline Forest & \multicolumn{5}{|c|}{ Global Hypothesis } \\
\hline Marg. Нyp. & Mar $=\cdots=$ Aug & Apr $=\cdots=$ Aug & May $=\cdots=$ Aug & Jun $=\cdots=$ Aug & Jul=Aug \\
\hline$H_{0, j}^{(\ell)}$ & $P\left\{R_{j}^{(\text {Mar })}<r_{j}^{(\text {Mar })}\right\}$ & $P\left\{R_{j}^{(A p r)}<r_{j}^{(A p r)}\right\}$ & $P\left\{R_{j}^{(\text {May })}<r_{j}^{(\text {May })}\right\}$ & $P\left\{R_{j}^{(J u n)}<r_{j}^{(J u n)}\right\}$ & $P\left\{R_{j}^{(J u l)}<r_{j}^{(j u l)}\right\}$ \\
\hline Apr $=$ Mar & 0.3925 & & & & \\
\hline May $=$ Apr & 0.5469 & 0.6235 & & & \\
\hline Jun = May & 0.4121 & 0.4085 & 0.4913 & & \\
\hline $\mathrm{Jul}=\mathrm{Jun}$ & 0.4229 & 0.4289 & 0.4629 & 0.4567 & \\
\hline Aug = Jul & 0.4116 & 0.4099 & 0.4095 & 0.4154 & 0.4523 \\
\hline$P\{Q<q\}$ & 0.3494 & 0.4218 & 0.4230 & 0.4108 & 0.4523 \\
\hline
\end{tabular}

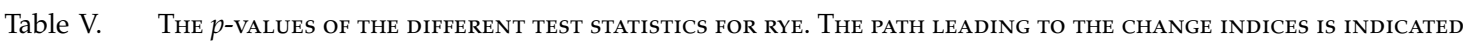

\begin{tabular}{|c|c|c|c|c|c|}
\hline Rye & \multicolumn{5}{|c|}{ Global Hypothesis } \\
\hline Marg. Hyp. & Mar $=\cdots=$ Aug & Apr $=\cdots=$ Aug & May $=\cdots=$ Aug & Jun $=\cdots=$ Aug & Jul=Aug \\
\hline$H_{0, j}^{(\ell)}$ & $P\left\{R_{j}^{(\text {Mar })}<r_{j}^{(\text {Mar })}\right\}$ & $P\left\{R_{j}^{(A p r)}<r_{j}^{(A p r)}\right\}$ & $P\left\{R_{j}^{(\text {May })}<r_{j}^{(\text {May })}\right\}$ & $P\left\{R_{j}^{(J u n)}<r_{j}^{(J u n)}\right\}$ & $P\left\{R_{j}^{(J u l)}<r_{j}^{(J u l)}\right\}$ \\
\hline Apr $=$ Mar & 0.4249 & & & & \\
\hline May $=$ Apr & 0.0026 & 0.0131 & & & \\
\hline Jun $=$ May & 0.1400 & 0.2599 & 0.3344 & & \\
\hline Jul = Jun & 0.0002 & 0.0023 & 0.0237 & 0.0242 & \\
\hline Aug = Jul & 0.1758 & 0.1292 & 0.0669 & 0.0468 & 0.0208 \\
\hline$P\{Q<q\}$ & 0.0000 & 0.0001 & 0.0011 & 0.0080 & 0.0208 \\
\hline
\end{tabular}

Table VI. The $p$-values of the Different test statistics For grass. The Path LEADing to the Change INDices IS INDiCATED

\begin{tabular}{|c|c|c|c|c|c|}
\hline \multirow{3}{*}{$\begin{array}{c}\text { Grass } \\
\text { Marg. Hyp. } \\
H_{0, j}^{(\ell)}\end{array}$} & \multicolumn{5}{|c|}{ Global Hypothesis } \\
\hline & Mar $=\cdots=$ Aug & Apr $=\cdots=$ Aug & May $=\cdots=$ Aug & Jun $=\cdots=$ Aug & Jul=Aug \\
\hline & $P\left\{R_{j}^{(\text {Mar })}<r_{j}^{(\text {Mar })}\right\}$ & $P\left\{R_{j}^{(A p r)}<r_{j}^{(A p r)}\right\}$ & $P\left\{R_{j}^{(\text {May })}<r_{j}^{(\text {May })}\right\}$ & $P\left\{R_{j}^{(J u n)}<r_{j}^{(j u n)}\right\}$ & $P\left\{R_{j}^{(J u l)}<r_{j}^{(j u l)}\right\}$ \\
\hline Apr $=$ Mar & 0.2808 & & & & \\
\hline May $=$ Apr & 0.0112 & 0.0638 & & & \\
\hline Jun = May & 0.0056 & 0.0319 & 0.1244 & & \\
\hline $\mathrm{Jul}=\mathrm{Jun}$ & 0.1094 & 0.2386 & 0.3879 & 0.4311 & \\
\hline Aug $=\mathrm{Jul}$ & 0.0000 & 0.0000 & 0.0000 & 0.0000 & 0.0000 \\
\hline$P\{Q<q\}$ & 0.0000 & 0.0000 & 0.0000 & 0.0000 & 0.0000 \\
\hline
\end{tabular}
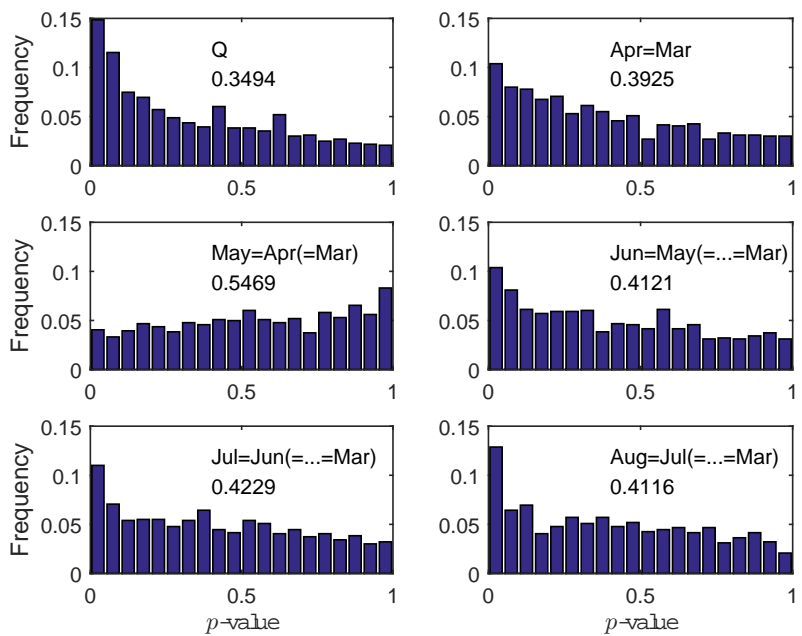

Figure 8. The histograms of the $p$-values for testing the hypotheses Mar $=\mathrm{Apr}=\mathrm{May}=\mathrm{Jun}=\mathrm{Jul}=\mathrm{Aug}$, i.e. no changes in the entire period, and for testing Apr = Mar, May = Apr (= Mar), Jun = May (= Apr = Mar), Jul = Jun (= May = Apr = Mar), and Aug = Jul $(=$ Jun = May = Apr = Mar) for the forest area. These histograms present the distribution of the pixelwise change indices for the forest area. The averages are found in Table IV. 

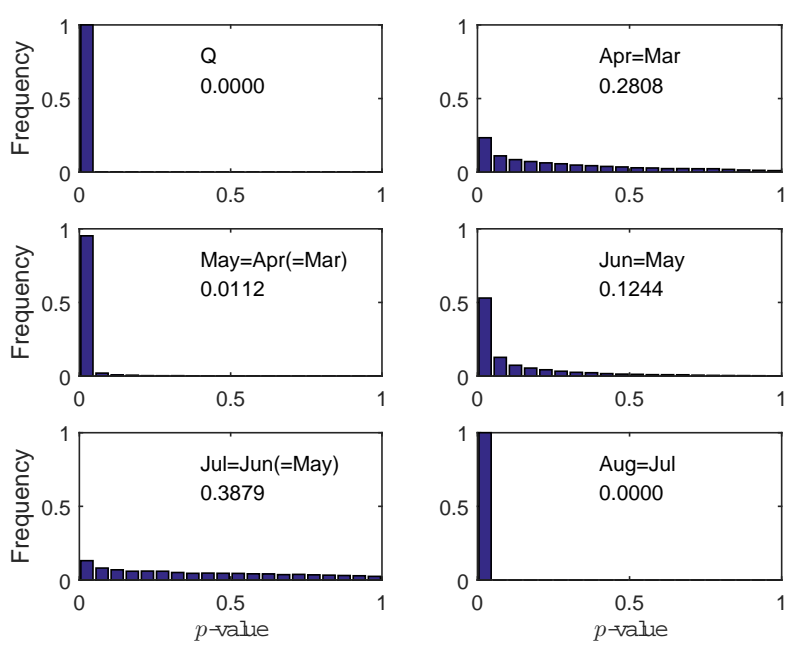

Figure 9. The histograms of the $p$-values for testing the hypotheses Mar $=\mathrm{Apr}=\mathrm{May}=\mathrm{Jun}=\mathrm{Jul}=\mathrm{Aug}$, i.e. no changes in the entire period, and for testing Apr = Mar, May = Apr (= Mar), Jun = May, Jul = Jun (= May), and Aug = Jul for the grass area. These histograms present the distribution of the pixelwise change indices for the grass area. The averages are found in Table VI.

\section{Imaging the Decomposed Test Statistics}

To give a visual impression of the results, Figures 10 and 11 show zooms onto the grass field considered in Table VI. Figure 10 shows images of the values of the different decomposed test statistics for the grass field. The first five rows show $-2 \rho_{j} \ln r_{j, 0 b s}$ for full polarimetry data stretched linearly between 0 and 100; first column for March, April, May, June, July and August; second column for April, May, June, July and August; third column for May, June, July and August; fourth column for June, July and August; fifth column for July and August. Row six shows the corresponding $-2 \rho \ln q_{\mathrm{obs}}$. The first images in all columns correspond to $-2 \rho \ln q_{\mathrm{obs}}$ for the pair-wise differences, i.e., the image in the first row, first column is $-2 \rho \ln q_{\text {obs }}$ in a $k=2$ analysis for the pair March and April, the image in the second row, first column is $-2 \rho \ln q_{\text {obs }}$ in a $k=2$ analysis for the pair April and May, the image in the third row, second column is $-2 \rho \ln q_{\mathrm{obs}}$ in a $k=2$ analysis for the pair May and June, the image in the fourth row, third column is $-2 \rho \ln q_{\mathrm{obs}}$ in a $k=2$ analysis for the pair June and July, and the image in the fifth row, fourth column is $-2 \rho \ln q_{\text {obs }}$ in a $k=2$ analysis for the pair July and August. Remember that if a change is detected at some point, the prerequisite for tests of later changes is no longer valid.

Figure 11 shows images of the $p$-values, i.e., the no-change probabilities corresponding to the test statistics in Figure 10 for the grass field. The values in the grass field in the center of the zoom images show clearly the same trend as indicated for the average values in Table VI. The arrows in both figures show the same change patterns as shown in Table VI and discussed in Section IV-A.

\section{Conclusions}

A test statistic for the equality of several covariance matrices following the complex Wishart distribution with an associated $p$-value has been presented. The test statistics is a direct generalization of a previously defined test statistics for pairwise comparison [22].

Using data from the airborne EMISAR system at L-band it is clearly shown how this test statistic is able to detect changes in a series of images, where the pairwise comparison fails to detect the changes.

After having detected that at least one change has occurred in a series of polarimetric SAR data, we have shown how one may decompose the likelihood ratio test statistic and thus obtain a procedure for determining the time points of change. Using data from the airborne Danish EMISAR system at L-band clearly shows that this procedure is able to identify the time points of change. The procedure may be extended to cope with other modes of SAR operations, such as the block-diagonal case, including azimuthally symmetric and diagonal-only data, see [22] for the situation with two time points.

\section{Appendix}

This appendix deals with the details of the likelihood ratio test statistics for comparing $k$ Gamma and $k$ complex Wishart distributions. Section A describes the Gamma case. The likelihood ratio test statistic is presented in Theorem 1, 

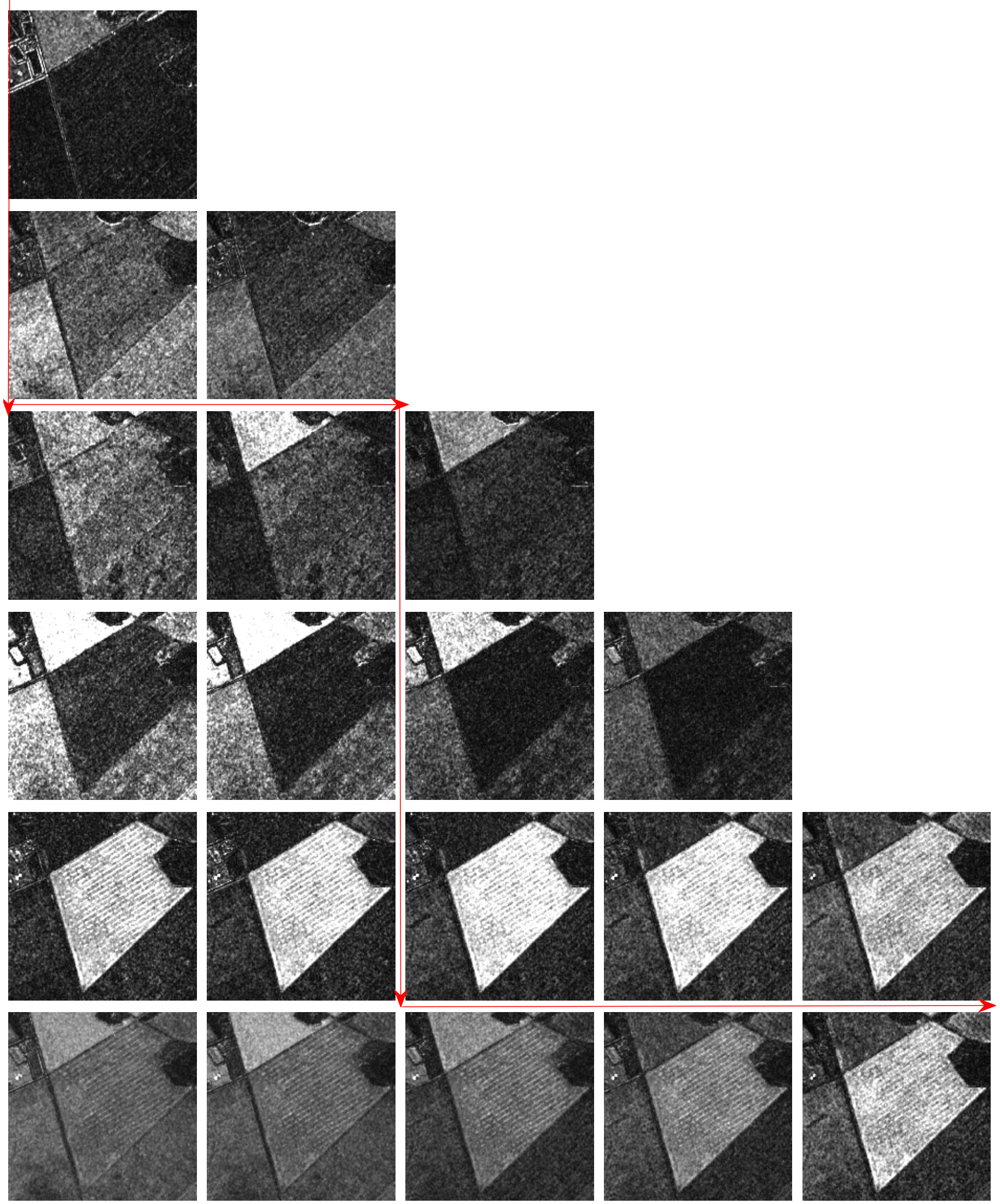

Figure 10. Zoom on grass field reported on in Table VI. The $p$-value corresponding to each subimage has the same relative position as in Table VI. Also, the path leading to the change indices is indicated in the figure as well as in the table. Rows $1-5:-2 \rho_{j} \ln r_{j, \text { obs }}$ for full polarimetry data stretched linearly between 0 and 100; first column for March, April, May, June, July and August; second column for April, May, June, July and August; third column for May, June, July and August; fourth column for June, July and August; fifth column for July and August. Last row: corresponding $-2 \rho \ln q_{\text {obs }}$ (first column stretched between 0 and 500, second column between 0 and 400, third column between 0 and 300 , fourth column between 0 and 200, and last column between 0 and 100). Dark areas correspond to no change. 

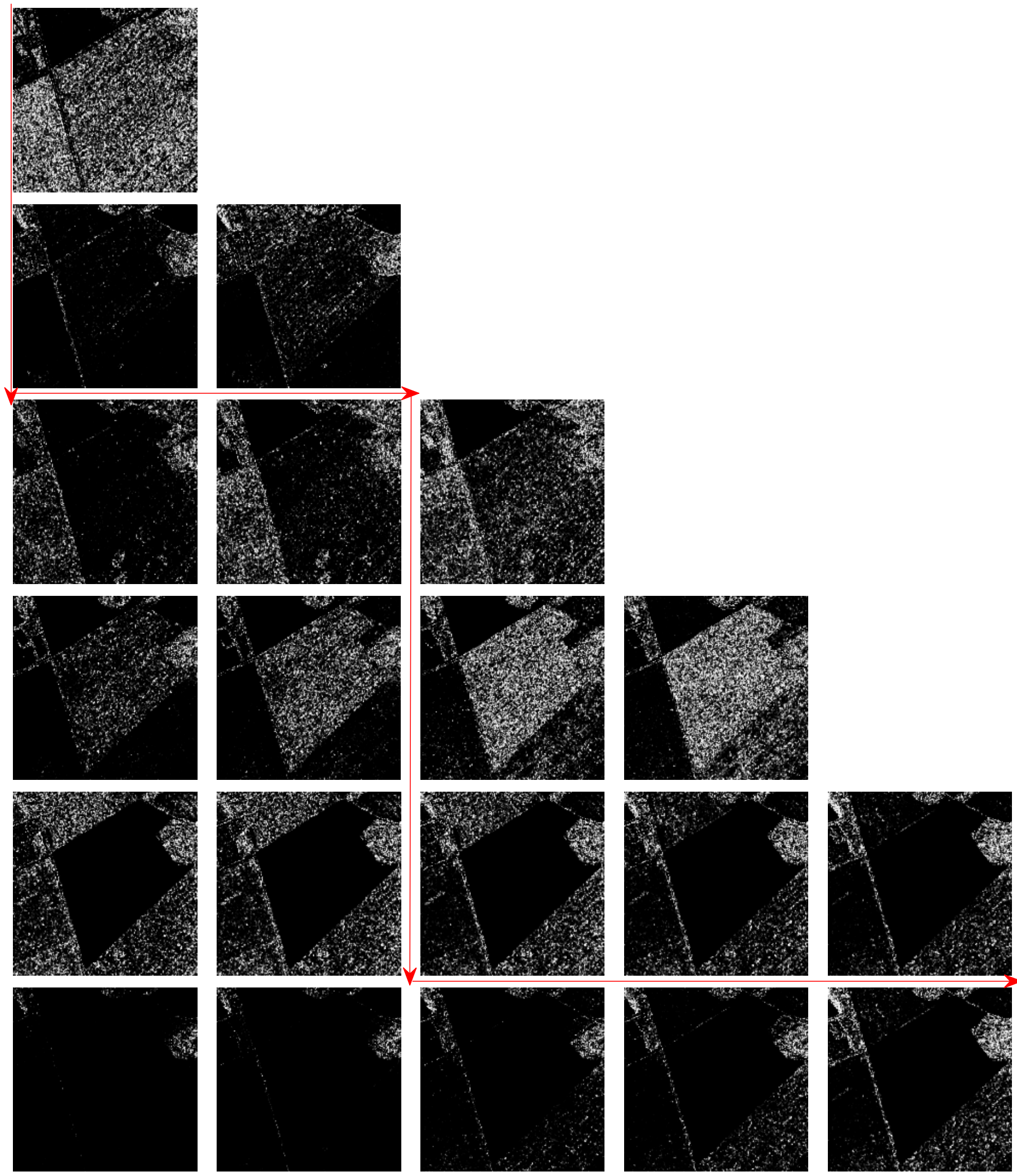

Figure 11. Zoom on grass field reported on in Table VI. The $p$-value corresponding to each subimage has the same relative position as in Table VI. Also, the path leading to the change indices is indicated in the figure as well as in the table. $p$-values, i.e., the no-change probability are stretched linearly between 0 and 1 . Dark areas correspond to change. 
and Theorem 2 gives the expected value of $Q^{h}$ which is used in providing the approximation formula in Theorem 3 using Box's method, [22], [35]. The lemma is a well known result on the independence of sum and a ratio of certain Gamma distributed random variables. This result is used in the proof of the (other) main result, namely the decomposition of the likelihood ratio test into the product of independent (under $H_{0}$ ) random variables $R_{j}$. The $R_{j} \mathrm{~S}$ provide tests for when a change actually occurs. Their distributions may be written as a function of independent Betadistributed random variables, and consequently the distribution of $Q$ may be written as a product of such variables (Theorem 5), [22], [35]. In Theorem 6 it is shown how we may use the F-distribution in testing instead of the $R_{i}$ s. This enables two-sided testing. The initial theorems are well known, the later ones are not (readily) available in the literature.

Section B addresses the same general problem as Section A but considering complex Wishart distributed random variables. Theorems 8,9 and 10 give the main results on the likelihood ratio test statistic, its expected values, and the large sample distribution. The second main result is the decomposition of $Q$ into independent components $R_{j}$ presented in Theorems 13 and 14, and the approximative distribution given in Theorem 15. We may apply the same philosophy with respect to mapping change patterns as was done in Section A. The results in the corollary in Section B is similar to the result in the corollary in Section A and may be used in finding other approximations to the distribution of $Q$.

\section{A. Comparing k Gamma Distributed Random Variables}

Theorem 1. We consider the independent Gamma distributed random variables

$$
X_{i} \sim G\left(n, \beta_{i}\right), i=1, \ldots, k
$$

where $n$ is a shape parameter and $\beta_{i}$ is a scale parameter; $\mathrm{E}\left\{X_{i}\right\}=n \beta_{i}$. We want to test the hypothesis $H_{0}$ against the alternative $H_{1}$ where

$$
H_{0}: \beta_{1}=\cdots=\beta_{k} \text { and } H_{1}: \exists i, j: \beta_{i} \neq \beta_{j} .
$$

Then the likelihood ratio test statistic for testing $H_{0}$ against the alternative $H_{1}$ is

$$
Q=\left\{k^{k} \frac{\prod_{i=1}^{k} X_{i}}{X^{k}}\right\}^{n} \text {. }
$$

Proof. Obtained by direct calculations.

Q.E.D.

Remark. In a one-sided analysis of variance (ANOVA, see for example [36]) with the same number of observations $f+1$ in each group, the unbiased estimators $s_{i}^{2}$ of the within group variances $\sigma_{i}^{2}$ follow chi-squared distributions with $f$ degrees of freedom, i.e.,

$$
s_{i}^{2} \sim \sigma_{i}^{2} \chi^{2}(f) / f, i=1, \ldots, k
$$

i.e.,

$$
f s_{i}^{2} \sim \sigma_{i}^{2} \chi^{2}(f)=G\left(\frac{f}{2}, 2 \sigma_{i}^{2}\right) .
$$

It now follows that the likelihood ratio test given above is equivalent to what is denoted Bartlett's test for homogeneity of variances in an ANOVA situation.

There is no simple closed form for the distribution of $Q$ in Theorem 1 but we may find large sample approximations to the distribution. First we state

Theorem 2. We consider the likelihood ratio test statistic $Q$ from Theorem 1 . Then we have $(h=1,2,3, \ldots)$

$$
\mathrm{E}\left\{Q^{h}\right\}=k^{k n h}\left(\frac{\Gamma(n(h+1))}{\Gamma(n)}\right)^{k} \frac{\Gamma(k n)}{\Gamma(k n(h+1))} .
$$

Proof. Obtained by direct calculations. 
In most cases it will be sufficient to approximate the distribution of $-2 \ln Q$ with a $\chi^{2}(f)$-distribution (under $H_{0}$ ). But we may use Box's approximation, [22], [35], to obtain the large sample distribution of $-\ln Q$. We have

Theorem 3. Let the situation be as in Theorem 1. Then we define

$$
\begin{aligned}
f & =k-1 \\
\rho & =1-\frac{k+1}{6 k n} \\
\omega_{2} & =-\frac{1}{4}(k-1)\left(1-\frac{1}{\rho}\right)^{2}
\end{aligned}
$$

and have

$$
\begin{aligned}
& P\{-2 \rho \ln Q \leq z\} \simeq P\left\{\chi^{2}(f) \leq z\right\} \\
& \quad+\omega_{2}\left[P\left\{\chi^{2}(f+4) \leq z\right\}-P\left\{\chi^{2}(f) \leq z\right\}\right] .
\end{aligned}
$$

Proof. Follows by straightforward calculations from Box's theorem by letting

$$
\xi_{\ell}=0, \eta_{j}=0, a=k, b=1, x_{\ell}=n, y_{j}=n k
$$

Q.E.D.

We now want to write the likelihood ratio test statistic $Q$ as a product of stochastically independent random variables (if $H_{0}$ is true). We start by introducing

Lemma. Let $X$ and $Y$ be independent and Gamma distributed $G(n, \beta)$ and $G(m, \beta)$ respectively. Then

$$
S=X+Y \text { and } U=\frac{X}{X+Y}
$$

are independent random variables, and $S$ follows a $G(n+m, \beta)$-distribution and $U$ a Beta-distribution, $B e(n, m)$.

Proof. Straightforward.

We now return to Gamma distributed random variables given in (27). We then have

Theorem 4. Let $\beta_{1}=\cdots=\beta_{j-1}$. Then the likelihood ratio test statistic for testing the hypothesis

$$
H_{0, j}: \beta_{j}=\beta_{1} \text { against } H_{1, j}: \beta_{j} \neq \beta_{1}
$$

is

$$
\begin{aligned}
R_{j} & =\frac{j^{j n}}{(j-1)^{(j-1) n}} \frac{\left(X_{1}+\cdots+X_{j-1}\right)^{(j-1) n} X_{j}^{n}}{\left(X_{1}+\cdots+X_{j}\right)^{j n}} \\
& =\frac{j^{j n}}{(j-1)^{(j-1) n}} U_{j}^{(j-1) n}\left(1-U_{j}\right)^{n}, j=2, \ldots, k
\end{aligned}
$$

where $U_{j}=S_{j-1} / S_{j}$ for $S_{j}=X_{1}+\cdots+X_{j}$. If all $\beta$ s are equal then $R_{2}, \ldots, R_{k}$ will be independent random variables.

Proof. The result on $R_{j}$ being the likelihood ratio test statistic follows immediately from Theorem 1 . According to Theorem $2 S_{j}$ and $U_{j}$ are independent. Therefore $U_{j}$ and $S_{j+1}=S_{j}+X_{j+1}$ will be independent and consequently $U_{j}$ and $U_{j+1}=S_{j} / S_{j+1}$ will be independent and the theorem follows.

Q.E.D.

Theorem 5. Let $\beta_{1}=\cdots=\beta_{k}$. Then we have

$$
Q=R_{2} \cdots R_{k}=k^{k n} \prod_{j=2}^{k} U_{j}^{(j-1) n}\left(1-U_{j}\right)^{n}
$$

where the $U_{j} \mathrm{~s}$ are independent and Beta-distributed

$$
U_{j} \sim B e((j-1) n, n) .
$$


Proof. Follows by direct computation and from Theorem 2 .

Q.E.D.

Theorem 6. The critical region for testing $H_{0, j}$ against $H_{1, j}$ is of the form

$$
R_{j} \leq c \Leftrightarrow \frac{X_{j}}{S_{j-1}} \leq c_{1} \text { or } \frac{X_{j}}{S_{j-1}} \geq c_{2}
$$

where $c_{1}$ and $c_{2}$ may be determined by realizing that

$$
(j-1) \frac{X_{j}}{S_{j-1}} \sim F(2 n, 2(j-1) n)
$$

if $H_{0, j}$ is true, and where $F$ stands for Fisher's $F$-distribution.

Proof. Straightforward.

B. Comparing $k$ Complex Wishart Distributed Random Variables

We start by stating three basic theorems (7, 8 and 9) on the likelihood ratio test statistic and its distribution.

Theorem 7. We consider independent random variables

$$
\boldsymbol{X}_{i} \sim W_{\mathbb{C}}\left(p, n, \boldsymbol{\Sigma}_{i}\right), i=1, \ldots, k .
$$

We wish to test the hypothesis

$$
H_{0}: \boldsymbol{\Sigma}_{1}=\cdots=\boldsymbol{\Sigma}_{k} \text { against } H_{1}: \exists i, j: \boldsymbol{\Sigma}_{i} \neq \boldsymbol{\Sigma}_{j} .
$$

Then the likelihood ratio test statistic is

$$
\begin{aligned}
Q & =k^{p n k} \frac{\prod_{i=1}^{k}\left|\boldsymbol{X}_{i}\right|^{n}}{|\boldsymbol{X}|^{n k}} \\
& =\left\{k^{p k} \frac{\prod_{i=1}^{k}\left|\boldsymbol{X}_{i}\right|}{|\boldsymbol{X}|^{k}}\right\}^{n} .
\end{aligned}
$$

where $\boldsymbol{X}=\boldsymbol{X}_{1}+\cdots+\boldsymbol{X}_{k}$.

Proof. Obtained by direct calculations.

Q.E.D.

Theorem 8. For the criterion $Q$ we have

$$
\mathrm{E}\left\{Q^{h}\right\}=k^{p k n h} \frac{\prod_{j=1}^{p} \Gamma(n k-j+1)}{\prod_{j=1}^{p} \Gamma(n k(1+h)-j+1)}\left\{\frac{\prod_{j=1}^{p} \Gamma(n(1+h)-j+1)}{\prod_{j=1}^{p} \Gamma(n-j+1)}\right\}^{k} .
$$

Using the multivariate Gamma function of the complex kind, see for example [37],

$$
\Gamma_{p}(n)=\pi^{p(p-1) / 2} \prod_{j=1}^{p} \Gamma(n-j+1)
$$

we get

$$
\mathrm{E}\left\{Q^{h}\right\}=k^{p k n h} \frac{\Gamma_{p}(n k)}{\Gamma_{p}(n k(1+h))}\left\{\frac{\Gamma_{p}(n(1+h))}{\Gamma_{p}(n)}\right\}^{k} .
$$

Proof. We consider independent $p$-dimensional random variables

$$
Y_{i j} \sim N_{\mathbb{C}}\left(\mu_{i}, \Sigma_{i}\right), i=1, \ldots, k, j=1, \ldots, n+1 .
$$


For $i=1, \ldots, k$ we introduce

$$
\boldsymbol{X}_{i}=\sum_{j=1}^{n+1}\left(Y_{i j}-\operatorname{ave}_{j}\left(Y_{i j}\right)\right)\left(Y_{i j}-\operatorname{ave}_{j}\left(Y_{i j}\right)\right)^{H}
$$

(the superscript $H$ denotes the complex conjugate transpose, and ave $e_{j}$ means the average over index $j$ ) and have the maximum likelihood estimators

$$
\hat{\boldsymbol{\Sigma}}_{i}=\frac{1}{n+1} \boldsymbol{X}_{i}
$$

with

$$
\boldsymbol{X}_{i} \sim W_{\mathbb{C}}\left(p, n, \Sigma_{i}\right)
$$

Setting $X=X_{1}+\cdots+X_{k}$ the test statistic for testing equality of the $\Sigma_{i}$ s becomes

$$
\begin{aligned}
\Lambda_{5} & =\left\{k^{p k} \frac{\prod_{i=1}^{k}\left|\frac{1}{n+1} \boldsymbol{X}_{i}\right|}{\left|\frac{1}{n+1} \boldsymbol{X}\right|^{k}}\right\}^{n+1} \\
& =\left\{k^{p k} \frac{\prod_{i=1}^{k}\left|\boldsymbol{X}_{i}\right|}{|\boldsymbol{X}|^{k}}\right\}^{n+1},
\end{aligned}
$$

see e.g. [38] p. 12. Comparing this with the likelihood ratio test criterion $Q$ in Theorem 4 we see that

$$
Q=\Lambda_{5}^{\frac{n}{n+1}} \text {. }
$$

Again following [38] we have

$$
\begin{aligned}
\mathrm{E}\left\{\Lambda_{5}\right\} & =k^{p k(n+1) t} \prod_{j=1}^{p} \frac{\Gamma(n k+1-j)}{\Gamma(n k+t j+(n+1) k t)} \prod_{j=1}^{k} \frac{\Gamma(n+1-j+(n+1) t)}{\Gamma(n+1-j)} \\
& =k^{p k(n+1) t} \frac{\Gamma_{p}(n k)}{\Gamma_{p}(n k+(n+1) k t)}\left\{\frac{\Gamma_{p}(n+(n+1) t)}{\Gamma_{p}(n)}\right\}^{k} .
\end{aligned}
$$

Therefore

$$
\begin{aligned}
\mathrm{E}\left\{Q^{h}\right\} & =\mathrm{E}\left\{\Lambda_{5}^{h \frac{n}{n+1}}\right\} \\
& =k^{p k n h} \frac{\Gamma_{p}(n k)}{\Gamma_{p}(n k+n k h)}\left\{\frac{\Gamma_{p}(n+n h)}{\Gamma_{p}(n)}\right\}^{k} .
\end{aligned}
$$

and the theorem follows.

Theorem 9. For $Q$ as in Theorems 4 and 5 we define

$$
\begin{aligned}
f & =(k-1) p^{2} \\
\rho & =1-\frac{\left(2 p^{2}-1\right)}{6(k-1) p}\left(\frac{k}{n}-\frac{1}{n k}\right) \\
\omega_{2} & =\frac{p^{2}\left(p^{2}-1\right)}{24 \rho^{2}}\left(\frac{k}{n^{2}}-\frac{1}{(n k)^{2}}\right)-\frac{p^{2}(k-1)}{4}\left(1-\frac{1}{\rho}\right)^{2}
\end{aligned}
$$

and have

$$
\begin{aligned}
& P\{-2 \rho \ln Q \leq z\} \simeq P\left\{\chi^{2}(f) \leq z\right\} \\
& \quad+\omega_{2}\left[P\left\{\chi^{2}(f+4) \leq z\right\}-P\left\{\chi^{2}(f) \leq z\right\}\right] .
\end{aligned}
$$


Proof. We rearrange the terms in $Q$ and obtain

$$
\begin{aligned}
E\left\{Q^{h}\right\} & =k^{p k n h} \frac{\Gamma_{p}(n k)}{\Gamma_{p}(n)^{k}} \frac{\Gamma_{p}(n(1+h))^{k}}{\Gamma_{p}(n k(1+h))} \\
& =k^{p k n h} \frac{\Gamma_{p}(n k)}{\Gamma_{p}(n)^{k}} \frac{\left\{\prod_{j=1}^{p} \Gamma(n(1+h)-j+1)\right\} \cdots\left\{\prod_{j=1}^{p} \Gamma(n(1+h)-j+1)\right\}}{\prod_{j=1}^{p} \Gamma(n k(1+h)-j+1)} .
\end{aligned}
$$

Letting

$$
\begin{array}{r}
a=k p, b=p \\
x_{\ell}=n, \ell=1, \ldots, k p \\
y_{j}=n k, j=1, \ldots, p \\
K=\frac{\Gamma_{p}(n k)}{\Gamma_{p}(n)^{k}},
\end{array}
$$

we have

$$
\left\{\prod_{j=1}^{p} y_{j}^{y_{j}}\right\} /\left\{\prod_{\ell=1}^{k p} x_{\ell}^{x_{\ell}}\right\}=(n k)^{p n k} / n^{n k p}=k^{n k p} .
$$

We define

$$
\eta_{j}=-j+1, j=1, \ldots, p
$$

and we let the $\xi_{\ell}$ s be $k$ versions of those numbers, i.e.,

$$
\begin{aligned}
\xi_{\ell} & =-\ell+1, \ell=1, \ldots, p \\
& \vdots \\
\xi_{\ell} & =-\ell+(k-1) p+1, \ell=(k-1) p+1, \ldots, k p .
\end{aligned}
$$

It is now obvious that we may use the result of Box [35] for example in the form given in [22] in approximating the distribution of $Q$. We have

$$
\begin{aligned}
f & =-2\left\{\sum_{\ell} \xi_{\ell}-\sum_{j} \eta_{j}-\frac{1}{2}(a-b)\right\} \\
& =-2\left\{k \sum_{\ell=1}^{p}(-\ell+1)-\sum_{j=1}^{p}(-j+1)-\frac{1}{2}(k p-p)\right\} \\
& =-2\left\{-\frac{1}{2}(k-1) p(p-1)-\frac{1}{2}(k-1) p\right\} \\
& =(k-1) p^{2} .
\end{aligned}
$$

Furthermore, for $\beta_{\ell}=(1-\rho) n$ and $\varepsilon_{j}=(1-\rho) n h$ we have

$$
\begin{aligned}
\omega_{1} & =\frac{1}{2}\left\{\sum_{\ell=1}^{p} \frac{k}{n \rho} B_{2}((1-\rho) n-\ell+1)-\sum_{j=1}^{p} \frac{1}{\rho n k} B_{2}((1-\rho) n k-j+1)\right\} \\
& =\frac{1}{2 \rho}\left\{k \sum_{\ell=1}^{p} \frac{1}{n} B_{2}((1-\rho) n-\ell+1)-\sum_{j=1}^{p} \frac{1}{n k} B_{2}((1-\rho) n k-j+1)\right\} .
\end{aligned}
$$

Now, for $s=n$ or $s=n k$ we have (straightforward calculations)

$$
\frac{1}{s} B_{2}((1-\rho) s-j+1)=(1-\rho)^{2} s-2(1-\rho) B_{1}(j)+\frac{1}{s} B_{2}(j) .
$$


The sums of the Bernoulli polynomials are

$$
\sum_{j=1}^{p} B_{1}(j)=\frac{1}{2} p^{2}, \sum_{j=1}^{p} B_{2}(j)=\frac{1}{6} p\left(2 p^{2}-1\right), \sum_{j=1}^{p} B_{3}(j)=\frac{1}{4} p^{2}\left(p^{2}-1\right) .
$$

Therefore

$$
\begin{aligned}
2 \rho \omega_{1}= & k\left\{p(1-\rho)^{2} n-2(1-\rho) \frac{1}{2} p^{2}+\frac{1}{6 n} p\left(2 p^{2}-1\right)\right\} \\
& -\left\{p(1-\rho)^{2} n k-2(1-\rho) \frac{1}{2} p^{2}+\frac{1}{6 n k} p\left(2 p^{2}-1\right)\right\} \\
= & -(k-1)(1-\rho) p^{2}+\frac{1}{6} p\left(2 p^{2}-1\right)\left(\frac{k}{n}-\frac{1}{n k}\right) .
\end{aligned}
$$

If we put $\omega_{1}=0$ we obtain

$$
(k-1)(1-\rho) p^{2}=\frac{1}{6} p\left(2 p^{2}-1\right)\left(\frac{k}{n}-\frac{1}{n k}\right)
$$

or

$$
(1-\rho)=\frac{1}{6(k-1) p}\left(2 p^{2}-1\right)\left(\frac{k}{n}-\frac{1}{n k}\right)
$$

For this value of $\rho$ we want to determine $\omega_{2}$. We have

$$
-6 \rho^{2} \omega_{2}=k \sum_{\ell=1}^{p} \frac{1}{n^{2}} B_{3}((1-\rho) n-\ell+1)-\sum_{j=1}^{p} \frac{1}{(n k)^{2}} B_{3}((1-\rho) n k-j+1) .
$$

Since

$$
\frac{1}{s^{2}} B_{3}((1-\rho) s-j+1)=s(1-\rho)^{3}-3(1-\rho)^{2} B_{1}(j)+\frac{1}{s}(1-\rho) 3 B_{2}(j)-\frac{1}{s^{2}} B_{3}(j)
$$

we get

$$
-6 \rho^{2} \omega_{2}=\frac{3}{2}(k-1) p^{2}(1-\rho)^{2}-\frac{1}{4} p^{2}\left(p^{2}-1\right)\left(\frac{k}{n^{2}}-\frac{1}{(n k)^{2}}\right)
$$

or

$$
\omega_{2}=-(k-1) \frac{p^{2}}{4}\left(1-\frac{1}{\rho}\right)^{2}+\frac{1}{24 \rho^{2}} p^{2}\left(p^{2}-1\right)\left(\frac{k}{n^{2}}-\frac{1}{(n k)^{2}}\right)
$$

and the theorem follows.

Coelho et al. [38] give other approximations to the distribution of expressions like $Q$.

Q.E.D.

In order to decompose the likelihood ratio criterion into independent components showing where possible changes may take place we need some auxiliary results on distributions of complex matrices.

Lemma. Let the independent random variables $X_{1}$ and $X_{2}$ be complex Wishart distributed

$$
\boldsymbol{X}_{i} \sim W_{\mathbb{C}}\left(p, n_{i}, \Sigma\right), i=1,2 .
$$

Let

$$
C=\left(X_{1}+X_{2}\right)^{-1 / 2}
$$

be a matrix so that

$$
C\left(X_{1}+X_{2}\right) C^{H}=I
$$

Then

$$
\begin{aligned}
& X_{1}+X_{2} \text { and } \\
& C X_{2} C^{H}=\left(X_{1}+X_{2}\right)^{-1 / 2} X_{2}\left\{\left(X_{1}+X_{2}\right)^{-1 / 2}\right\}^{H}
\end{aligned}
$$


are independent.

Proof. First we consider random variables $\boldsymbol{U}_{i}, i=1,2$ that are Wishart distributed with $\Sigma=I$. In this case the joint density is

$$
f\left(\boldsymbol{u}_{1}, \boldsymbol{u}_{2}\right)=K \exp \left(-\operatorname{trace}\left(\boldsymbol{u}_{1}+\boldsymbol{u}_{2}\right)\right)\left|\boldsymbol{u}_{1}\right|^{n_{1}-p}\left|\boldsymbol{u}_{2}\right|^{n_{2}-p} .
$$

In this case the theorem follows directly from, e.g., [39]. The general case now follows by considering the transformation given by

$$
\boldsymbol{X}_{i}=\Sigma^{1 / 2} \boldsymbol{U}_{i}\left(\Sigma^{1 / 2}\right)^{H}
$$

We then obtain

$$
\begin{aligned}
\boldsymbol{I} & =C\left(\boldsymbol{X}_{1}+X_{2}\right) C^{H} \\
& =C \Sigma^{1 / 2}\left(\boldsymbol{U}_{1}+\boldsymbol{U}_{2}\right)\left(C \Sigma^{1 / 2}\right)^{H} \\
C \boldsymbol{X}_{2} C^{H} & =C \Sigma^{1 / 2} \boldsymbol{U}_{2}\left(C \Sigma^{1 / 2}\right)^{H} .
\end{aligned}
$$

Thus, the previous results applies to $\boldsymbol{U}_{1}, \boldsymbol{U}_{2}$ and the theorem follows.

Q.E.D.

Returning to the likelihood ratio statistic $Q$ we can now prove

Theorem 10. Given that

$$
\Sigma_{1}=\Sigma_{2}=\cdots=\Sigma_{j-1}
$$

then the likelihood ratio test statistic $R_{j}$ for testing the hypothesis

$$
H_{0}: \boldsymbol{\Sigma}_{j}=\boldsymbol{\Sigma}_{j-1} \text { against } H_{1}: \boldsymbol{\Sigma}_{j} \neq \boldsymbol{\Sigma}_{j-1}
$$

is

$$
R_{j}=\frac{j^{j p n}}{(j-1)^{(j-1) p n}} \frac{\left|\boldsymbol{X}_{1}+\cdots+\boldsymbol{X}_{j-1}\right|^{(j-1) n}\left|\boldsymbol{X}_{j}\right|^{n}}{\left|\boldsymbol{X}_{1}+\cdots+\boldsymbol{X}_{j}\right|^{j n}} .
$$

Furthermore

$$
Q=\prod_{j=2}^{k} R_{j}
$$

Proof. Straightforward.

Theorem 11. If the hypothesis

$$
H_{0}: \Sigma_{1}=\cdots=\Sigma_{k}
$$

is true and $n \geq p$, then the quantities $R_{2}, \ldots, R_{k}$ are independent.

Proof. We find $C_{j}$ so that

$$
C_{j}\left(X_{1}+\cdots+X_{j}\right) C_{j}^{H}=I
$$

and put

$$
E_{j}=C_{j}\left(X_{1}+\cdots+X_{j-1}\right) C_{j}^{H}
$$

getting

$$
I-E_{j}=C_{j} X_{j} C_{j}^{H}
$$

From the above lemma it follows that $\boldsymbol{E}_{j}$ is independent of $\boldsymbol{X}_{1}+\cdots+\boldsymbol{X}_{j}$ and therefore also independent of $\boldsymbol{X}_{1}+\cdots+$ $\boldsymbol{X}_{j}+\boldsymbol{X}_{j+1}$. This implies that

$$
E_{j} \text { and } C_{j+1}\left(X_{1}+\cdots+X_{j}\right) C_{j+1}^{H}=E_{j+1}
$$


are independent. Arguing along those lines gives the theorem.

Q.E.D.

Corollary. The quantities $R_{j}$ and $Q$ may be written

$$
\begin{aligned}
R_{j} & =\frac{j^{j p n}}{(j-1)^{(j-1) p n}}\left|\boldsymbol{E}_{j}\right|^{(j-1) n}\left|\boldsymbol{I}-\boldsymbol{E}_{j}\right|^{n} \\
Q & =k^{k p n} \prod_{j=2}^{k}\left|\boldsymbol{E}_{j}\right|^{(j-1) n}\left|\boldsymbol{I}-\boldsymbol{E}_{j}\right|^{n} .
\end{aligned}
$$

Proof. Straightforward.

Q.E.D.

We conclude this section by stating a theorem on the distribution of the components $R_{j}$ in the decomposition of the likelihood ratio citerion.

Theorem 12. Let the situation be as in Theorem 10. Letting

$$
\begin{aligned}
f & =p^{2} \\
\rho_{j} & =1-\frac{2 p^{2}-1}{6 p n}\left(1+\frac{1}{j(j-1)}\right) \\
\omega_{2 j} & =-\frac{p^{2}}{4}\left(1-\frac{1}{\rho_{j}}\right)^{2}+\frac{1}{24 n^{2}} p^{2}\left(p^{2}-1\right)\left(1+\frac{2 j-1}{j^{2}(j-1)^{2}}\right) \frac{1}{\rho_{j}^{2}} .
\end{aligned}
$$

Then

$$
\begin{aligned}
& P\left\{-2 \rho_{j} \ln R_{j} \leq z\right\} \simeq P\left\{\chi^{2}(f) \leq z\right\} \\
& +\omega_{2 j}\left[P\left\{\chi^{2}(f+4) \leq z\right\}-P\left\{\chi^{2}(f) \leq z\right\}\right] .
\end{aligned}
$$

Proof. Follows from the two-sample test for equality of complex Wishart matrices.

Q.E.D.

Coelho et al. [38] give other approximations to the distribution of the expression like $R_{j}$.

\section{REFERENCES}

[1] R. Touzi, A. Lopes, and P. Bousquet, "A statistical and geometrical edge detector for SAR images," IEEE Transactions on Geoscience and Remote Sensing, vol. 26, pp. 764-773, 1988.

[2] E. J. Rignot and J. J. Van Zyl, "Change detection techniques for ERS-1 SAR data," IEEE Transactions on Geoscience and Remote Sensing, vol. 31, no. 4, pp. 896-906, 1993.

[3] Y. Bazi, L. Bruzzone, and F. Melgani, "An unsupervised approach based on the generalized Gaussian model to automatic change detection in multitemporal SAR images," IEEE Transactions on Geoscience and Remote Sensing, vol. 43, no. 4, pp. 874-887, 2005.

[4] G. Moser and S. B. Serpico, "Generalized minimum-error thresholding for unsupervised change detection from SAR amplitude imagery," IEEE Transactions on Geoscience and Remote Sensing, vol. 44, no. 10, pp. 2972-2982, 2006.

[5] Y. Ban and O. A. Yousif, "Multitemporal spaceborne SAR data for urban change detection in China," IEEE Journal of Selected Topics in Applied Earth Observations and Remote Sensing, vol. 5, no. 4, pp. 1087-1094, 2012.

[6] H. Hu and Y. Ban, "Unsupervised change detection in mulitemporal SAR images over large urban areas," IEEE Journal of Selected Topics in Applied Earth Observations and Remote Sensing, vol. 7, no. 8, pp. 3248-3261, 2014.

[7] C. Carincotte, S. Derrode, and S. Bourennane, "Unsupervised change detection on SAR images using fuzzy hidden Markov chains," IEEE Transactions on Geoscience and Remote Sensing, vol. 44, no. 2, pp. 432-441, 2006.

[8] F. Bovolo and L. Bruzzone, "A detail-preserving scale-driven approach to change detection in multitemporal SAR images," IEEE Transactions on Geoscience and Remote Sensing, vol. 43, no. 12, pp. 2963-2972, 2005.

[9] T. Celik, "A Bayesian approach to unsupervised multiscale change detection in synthetic aperture radar images," Signal Processing, vol. 90, no. 5, pp. 1471-1485, 2010.

[10] M. Gong, Z. Zhou, and J. Ma, "Change detection in synthetic aperture radar images based on image fusion and fuzzy clustering," IEEE Transactions on Image Processing, vol. 21, no. 4, pp. 2141-2151, 2012.

[11] F. Bovolo, C. Martin, and L. Bruzzone, "A hierarchical approach to change detection in very high resolution SAR images for surveillance applications," IEEE Transactions on Geoscience and Remote Sensing, vol. 51, no. 4, pp. 2042-2054, 2013. 
[12] P. Gamba, F. Dell'Acqua, and G. Lisini, "Change detection of multitemporal SAR data in urban areas combining feature-based and pixel-based techniques," IEEE Transactions on Geoscience and Remote Sensing, vol. 44, no. 10, pp. 2820-2827, 2006.

[13] J. Inglada and G. Mercier, "A new statistical similarity measure for change detection in multitemporal SAR images and its extension to multiscale change analysis," IEEE Transactions on Geoscience and Remote Sensing, vol. 45, no. 5, pp. 1432-1445, 2007.

[14] F. Chatelain, J.-Y. Tourneret, and J. Inglada, "Change detection in multisensory SAR images using bivariate Gamma distributions," IEEE Transactions on Image Processing, vol. 17, no. 3, pp. 249-258, 2008.

[15] C. Pratola, F. Del Frate, G. Schiavon, and D. Solimini, "Toward fully automatic detection of changes in suburban areas from VHR SAR images by combining multiple neural-network modes," IEEE Transactions on Geoscience and Remote Sensing, vol. 51, no. 4, pp. 2055-2066, 2013.

[16] S. Hachicha and F. Chaabane, "On the SAR change detection review and optimal decision," International Journal of Remote Sensing, vol. 35, no. 5, pp. 1693-1714, 2014.

[17] O. Yousif and Y. Ban, "Improving SAR-based urban change detection by combining MAP-MRF classifier and nonlocal means similarity weights," IEEE Journal of Selected Topics in Applied Earth Observations and Remote Sensing, vol. 7, no. 10, pp. 4288-4300, 2014.

[18] W. Dierking and H. Skriver, "Change detection for thematic mapping by means of airborne multitemporal polarimetric SAR imagery," IEEE Transactions on Geoscience and Remote Sensing, vol. 40, pp. 618-636, 2002.

[19] Q. Qong, "Polarization state conformation and its application to change detection in polarimetric SAR data," IEEE Geoscience and Remote Sensing Letters, vol. 1, no. 4, pp. 304-307, 2004.

[20] G. Moser, S. B. Serpico, and G. Vernazza, “Unsupervised change detection from multichannel SAR images," IEEE Geoscience and Remote Sensing Letters, vol. 4, no. 2, pp. 278-282, 2007.

[21] G. Moser and S. B. Serpico, "Unsupervised change detection from multichannel SAR data by Markovian data fusion," IEEE Transactions on Geoscience and Remote Sensing, vol. 47, no. 7, pp. 2114-2128, 2009.

[22] K. Conradsen, A. A. Nielsen, J. Schou, and H. Skriver, "A test statistic in the complex Wishart distribution and its application to change detection in polarimetric SAR data," IEEE Transactions on Geoscience and Remote Sensing, vol. 41, no. 1, pp. 4-19, Jan. 2003, http://www.imm.dtu.dk/pubdb/p.php?1219.

[23] P. Formont, F. Pascal, G. Vasile, J. P. Ovarlez, and L. Ferro-Famil, "Statistical classification for heterogeneous polarimetric SAR images," IEEE Journal of Selected Topics in signal Processing, vol. 5, no. 3, pp. 567-576, 2011.

[24] A. Marino, S. Cloude, and J. Sanchez-Lopez, "A new polarimetric change detector in radar imagery," IEEE Transactions on Geoscience and Remote Sensing, vol. 51, no. 5, pp. 2986-3000, 2013.

[25] V. Akbari, S. N. Anfinsen, A. P. Doulgeris, and T. Eltoft, "The Hotelling-Lawley trace statistic for change detection in polarimetric SAR data under the complex Wishart distribution," in IEEE International Geoscience and Remote Sensing Symposium (IGARSS), Melbourne, Victoria, Australia, 21-26 July 2013, pp. 4162-4165.

[26] M. Liu, H. Zhang, C. Wang, and F. Wu, "Change detection of multilook polarimetric SAR images using heterogeneous clutter models," IEEE Transactions on Geoscience and Remote Sensing, vol. 52, no. 12, pp. 7483-7494, 2014.

[27] W. T. Coombs, J. Algina, and D. O. Oltman, “Univariate and multivariate omnibus hypothesis tests selected to control type I error rates when population variances are not necessarily equal," Review of Educational Research, vol. 66, no. 2, pp. 137-179, 1996.

[28] A. A. Nielsen, K. Conradsen, and H. Skriver, "Change detection in full and dual polarization, single- and multi-frequency SAR data," IEEE Journal of Selected Topics in Applied Earth Observations and Remote Sensing, vol. 8, no. 8, pp. 4041-4048, Aug. 2015, http://www.imm.dtu.dk/pubdb/p.php?6827.

[29] J. J. van Zyl and F. T. Ulaby, "Scattering matrix representation for simple targets," in Radar Polarimetry for Geoscience Applications, F. T. Ulaby and C. Elachi, Eds. Artech, Norwood, MA, 1990.

[30] S. N. Madsen, E. L. Christensen, N. Skou, and J. Dall, “The Danish SAR system: Design and initial tests," IEEE Transactions on Geoscience and Remote Sensing, vol. 29, pp. 417-476, 1991.

[31] E. L. Christensen, N. Skou, J. Dall, K. Woelders, J. H. Jørgensen, J. Granholm, and S. N. Madsen, "EMISAR: An absolutely calibrated polarimetric L- and C-band SAR," IEEE Transactions on Geoscience and Remote Sensing, vol. 36, pp. 1852-1865, 1998.

[32] R. G. Miller Jr., Simultaneous Statistical Inference, Springer Verlag, second edition, 1981.

[33] T. W. Anderson, An Introduction to Multivariate Statistical Analysis, John Wiley, New York, third edition, 2003.

[34] S. R. Cloude and E. Pottier, "An entropy based classification scheme for land applications of polarimetric SAR," IEEE Transactions on Geoscience and Remote Sensing, vol. 35, no. 1, pp. 68-78, 1997.

[35] G. E. P. Box, "A general distribution theory for a class of likelihood criteria," Biometrika, vol. 36, pp. 317-346, 1949.

[36] G. James, D. Witten, T. Hastie, and R. Tibshirani, An Introduction to Statistical Learning with Applications in R, Springer, 2013.

[37] S. N. Anfinsen and T. Eltoft, "Application of the matrix-variate Mellin transform to analysis of polarimetric radar images," IEEE Transactions on Geoscience and Remote Sensing, vol. 49, no. 6, pp. 2281-2295, 2011.

[38] C. A. Coelho, F. J. Marques, and B. C. Arnold, "A general approach to the exact and near-exact distributions of the main likelihood ratio test statistics used in the complex multivariate Normal setting," Preprint, 2011, http://www.dm.fct.unl.pt/sites/www.dm.fct.unl.pt/files/preprints/2011/19_11.pdf.

[39] A. K. Gupta and D.G Kabe, "An n-variate characterization of the Gamma and the complex Wishart densities," Applied Mathematics Letters, vol. 10, no. 4, pp. 119-122, 1997. 


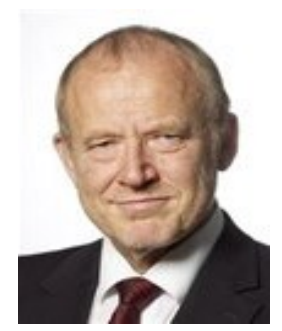

Knut Conradsen received the Cand. Scient. degree in mathematics in 1970 from the University of Copenhagen, Denmark. He has been with the Technical University of Denmark (DTU) since 1970. Presently he is Professor of Statistical Image Analysis at the Department of Applied Mathematics and Computer Science, DTU. From 1995 to 2010 he was Provost (Deputy Rector) of DTU. His main research interest is the application of statistics and statistical models in primarily medical image analysis, remote sensing and industrial applications. Work includes analysis of multi-/hyperspectral and multi-temporal data, as well from optical as from radar sensors.

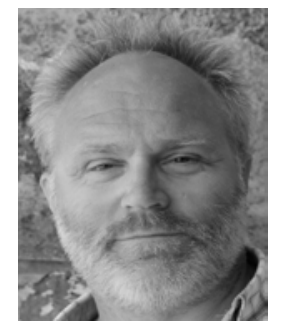

Allan Aasbjerg Nielsen received the M.Sc. degree from the Department of Electrophysics at the Technical University of Denmark (DTU), Lyngby, Denmark, in 1978 and the Ph.D. degree from Informatics and Mathematical Modelling (IMM), DTU, in 1994. He is currently an Associate Professor with the Department of Applied Mathematics and Computer Science, DTU. From 1977 to 1978, he was with the Danish Defense Research Establishment. From 1978 to 1985, he worked on energy conservation in housing with the Thermal Insulation Laboratory, DTU. He was with the section for image analysis from 1985 to 2001 and with the section for geoinformatics from 2001 to 2006, both at IMM. From 2007 to 2013, he was with the Danish National Space Center's section for geodesy. Since 1985, he has worked on several national and international projects on the development, implementation, and application of statistical methods, and remote sensing in mineral exploration, mapping, geology, agriculture, environmental monitoring, oceanography, geodesy, and security funded by industry, the European Union, Danida (the Danish International Development Agency), and the Danish National Research Councils.

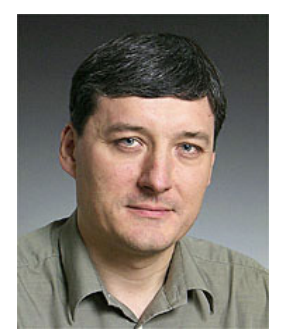

Henning Skriver received the M.Sc. and Ph.D. degrees in electrical engineering from the Technical University of Denmark (DTU), Lyngby, Denmark, in 1983 and 1989, respectively. Since 1983, he has been with the DTU, where he is an Associate Professor, the Head of Microwaves and Remote Sensing, and Deputy Head of Department at DTU Space. His work has primarily been concerned with topics related to the utilization of synthetic aperture radar (SAR) data for different applications, such as sea ice parameter retrieval from SAR data, as well as different aspects of land applications of SAR data, such as forestry, agricultural, environmental, and topographic mapping applications using both satellite SAR data and data from polarimetric SARs, e.g., the Danish airborne polarimetric SAR, EMISAR, and the German experimental SAR ESAR. His interests also include methods for the processing of SAR data; SAR image simulation; SAR image filtering; speckle statistics; and texture analysis, segmentation, calibration, change detection, classification, and polarimetric analysis and processing. 